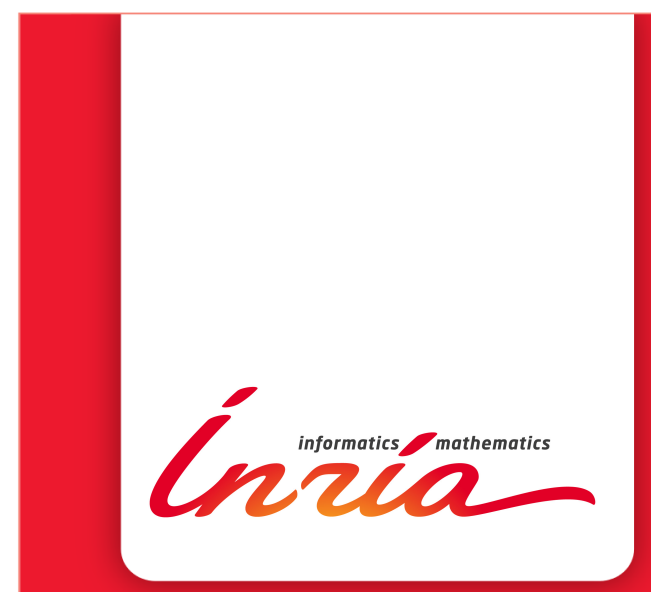

"Classical"

Electropermeabilization

Modeling at the Cell

Scale

O. Kavian ${ }^{1}$, M. Leguèbe ${ }^{2}$, C. Poignard ${ }^{2}$, L. Weynans ${ }^{2}$

${ }^{1}$ Département de Mathématiques, LMV CNRS UMR8100,

Université Versailles-Saint-Quentin-en-Yvelines, France

${ }^{2}$ Team MC2, INRIA Bordeaux-Sud-Ouest \& CNRS UMR 5251,

Université de Bordeaux, France

RESEARCH

REPORT

$\mathrm{N}^{\circ} 8005$

June 2012

Project-Teams MC2 



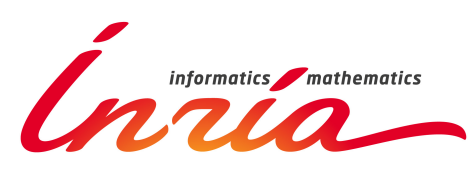

\title{
"Classical" Electropermeabilization Modeling at the Cell Scale
}

\author{
O. Kavian ${ }^{1}$, M. Leguèbe ${ }^{2}$, C. Poignard ${ }^{2 *}$ L. Weynans ${ }^{2}$ \\ ${ }^{1}$ Département de Mathématiques, LMV CNRS UMR8100, \\ Université Versailles-Saint-Quentin-en-Yvelines, France \\ ${ }^{2}$ Team MC2, INRIA Bordeaux-Sud-Ouest \& CNRS UMR 5251, \\ Université de Bordeaux, France \\ Project-Teams MC2
}

Research Report n8005 - June 2012 - 30 pages

\begin{abstract}
In this paper, we derive two models (a static and a dynamical model) based on the description of the electric potential in a biological cell in order to model the cell electropermeabilization. Existence and uniqueness results are provided for each model, and an accurate numerical method to compute the solution is described. We then present numerical results that corroborate the experimental results, which tends to justify the validity of our modeling. We emphasize that our new models involve very few parameters, compared with the most achieved model but they describe the same qualitative results. Moreover our numerical results are quantitavely close to the experimental data.
\end{abstract}

Key-words: Cell modeling, Non-linear partial differential equations, Finite differences on cartesian grids

* Corresponding author: clair.poignard@inria.fr

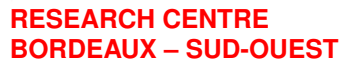

351, Cours de la Libération

Bâtiment A 29

33405 Talence Cedex 


\section{Modélisation de l'électroporation cellulaire par micropulses}

Résumé : Dans cet article, deux modèles d'électroporation cellulaire sont présentés: un modèle statique et un modèle dynamique. Ces modèles sont obtenus via une description du potentiel électrique dans une cellule. Des résultats d'existence et d'unicité sont donnés pour chaque modèle et une méthode numérique permettant de calculer précisément la solution de chacun de ces problèmes est décrite. Des simulations numériques qui corroborent les résultats expérimentaux concluent ce travail, justifiant la validité de la modélisation. Notons que ces nouveaux modèles ne comportent que peu de paramètres, comparés aux modèles existants, tout en décrivant les mêmes comportements, avec des résultats proches des résultats expérimentaux.

Mots-clés : Modélisation bio-cellulaire, Equations aux dérivées partielles non linéaires, Méthodes de différences finies sur grilles cartésiennes 


\section{Contents}

\begin{tabular}{lll}
\hline & Introduction & 4 \\
\hline
\end{tabular}

$1.1 \quad$ Electric potential in a biological cell . . . . . . . . . . . . . . . . . . . . . 4

1.2 Electropermeabilization phenomenon . . . . . . . . . . . . . . . . . 5

1.3 Modeling principle $\ldots \ldots \ldots \ldots \ldots \ldots$

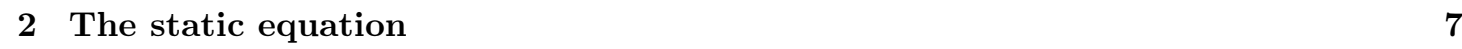

2.1 Existence and uniqueness of the static potential . . . . . . . . . . . . . . . . . . 7

\begin{tabular}{|lll}
\hline 3 & The dynamical model & 11
\end{tabular}

3.1 Heuristics of the modeling . . . . . . . . . . . . . . . . . . . . 11

3.2 Statement of the mathematical problem . . . . . . . . . . . . . . . . . . . 12

3.3 Properties of the function $X$. . . . . . . . . . . . . . . . . . . . . . 13

3.4 Existence and uniqueness of the dynamical potential . . . . . . . . . . . . . . . . 14

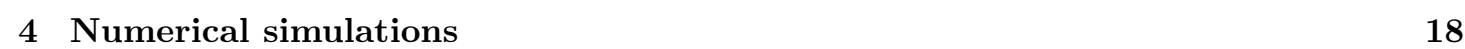

4.1 Spatial discretization . . . . . . . . . . . . . . . . . . . . . . . . . 18

4.2 Accuracy of the finite difference method . . . . . . . . . . . . . . . . . . . . 19

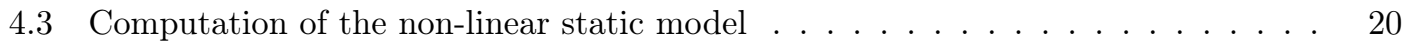

4.3 .1 Influence of the parameter $S_{\text {ir }} \ldots \ldots \ldots \ldots$. . . . . . . . . . . . . . . . . . . 22

4.3.2 Comparison with the model of Ivorra, Mir and Villemejeane . . . . . . . . 22

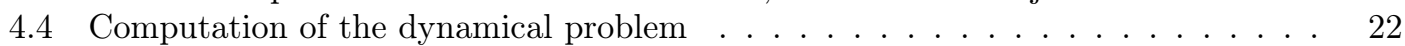

4.4 .1 Time-discretization of the model . . . . . . . . . . . . . . . . . . . . 22

4.4 .2 Main parameters influence . . . . . . . . . . . . . . 25

4.4.3 Comparison with the model of Neu, Krassowska, and Debruin . . . . . . . 25

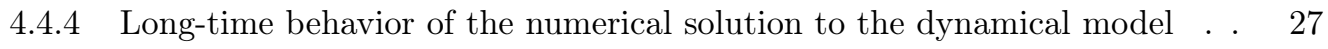

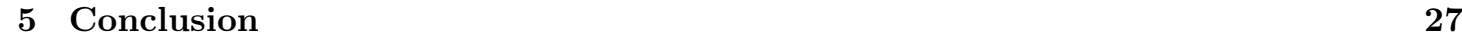




\section{Introduction}

The distribution of the electric potential in a biological cell is important for bio-electromagnetic investigations. A sufficiently large magnitude of the difference of transmembrane potential (denoted from now on by $\triangle \mathrm{TMP}$ ), which is the difference of the electric potentials between both sides of the cell membrane, leads to an increase of the membrane permeability [19, 23. Molecules such as bleomycin can then diffuse across the plasma membrane. This phenomenon, called electropermeabilization, has already been used in oncology and holds promises in gene therapy [11, 20, motivating precise assessments of the $\Delta \mathrm{TMP}$.

In this paper, we aim at studying theoretically and numerically two non-linear electrical models (a static and a dynamical model) of biological cells. These models are inspired from the static model of Ivorra et al. in [10. They describe the behavior of both electric potential and membrane conductivity when the cell is submitted to an electric pulse through a few parameters that will be fitted with the experiments. The inverse problem solving to calibrate the models, which is the main goal of this research, is not tackled in the present paper. This article is a first step, in which we present, and study theoretically and numerically new models with a few parameters, in order to simplify the forthcoming inverse problem solving. We emphasize these models are phenomenological in the sense that the membrane conductivity is described by an ad hoc law, which does not come from an homogenization of the nanoscale phenomena. Before stating the model we are going to study, we now detail the notation of the paper.

Notation 1.1. Throughout this article, we shall use the following conventions and notation:

- We generically denote by $\mathbf{n}$ the normal to a closed smooth surface of $\mathbb{R}^{3}$ (or a curve of $\mathbb{R}^{2}$ ) outwardly directed from the inside to the outside of the domain enclosed by the surface.

- Let $\mathcal{C}$ be a surface embedded in $\mathbb{R}^{3}$, and let $u$ be a sufficiently smooth function (in an appropriate sense) defined in a tubular neighborhood of $\mathcal{C}$. We define $u_{\left.\right|_{\mathcal{C}^{ \pm}}}$by

$$
\forall x \in \mathcal{C}, \quad u_{\left.\right|_{\mathcal{C}^{ \pm}}}(x)=\lim _{\tau \rightarrow 0^{+}} u(x \pm \tau \mathbf{n}(x)) .
$$

The notation $\partial_{\mathbf{n}} u_{\left.\right|_{\mathcal{C}^{ \pm}}}$and $\partial_{\mathbf{t}} u_{\left.\right|_{\mathcal{C}^{ \pm}}}$stands for the normal and the tangential components of $\nabla u$ :

$$
\begin{aligned}
\forall x \in \mathcal{C}, \quad \partial_{\mathbf{n}} u_{\left.\right|_{\mathcal{C}^{ \pm}}}(x) & =\lim _{\tau \rightarrow 0^{+}} \nabla u(x \pm \tau \mathbf{n}(x)) \cdot \mathbf{n}(x), \\
\partial_{\mathbf{t}} u_{\left.\right|_{\mathcal{C}^{ \pm}}}(x) & =\nabla u(x)-\partial_{\mathbf{n}} u(x) \mathbf{n}(x)
\end{aligned}
$$

where the dot ". " denotes the Euclidean scalar product of $\mathbb{R}^{3}$. In the case of $\mathbb{R}^{2}$, the analogous notation is easily adapted.

- The jump $[u]_{\mathcal{C}}$ of a function $u$ defined in a neighborhood of the surface $\mathcal{C}$ is defined by

$$
[u]_{\mathcal{C}}=\left.u\right|_{\mathcal{C}^{+}}-\left.u\right|_{\mathcal{C}^{-}}
$$

\subsection{Electric potential in a biological cell}

A biological cell is a high contrast medium composed of a conducting cytoplasm $\mathcal{O}_{\text {c }}$ surrounded by a thin and very insulating layer, embedded in a bath $\mathcal{O}_{\mathrm{e}}$ (see Fig. 1). The plasma membrane is a phospholipid bilayer, which is sprinkled over with proteins. Due to its thickness and its electrical properties, the membrane can be modeled as a surface electric material $\Gamma$ with a capacity $C_{\mathrm{m}}$ and a surface conductivity $S_{\mathrm{m}}$. We refer to the seminal articles of Hodgkin, Goldman, Katz, et 
al. for the electric description of cell membranes [5, 7, 9, 8]. Let $\sigma$ be the conductivity of the medium, that is

$$
\sigma= \begin{cases}\sigma_{\mathrm{e}}, & \text { in the exterior domain } \mathcal{O}_{\mathrm{e}} \\ \sigma_{\mathrm{c}}, & \text { inside the cell } \mathcal{O}_{\mathrm{c}} .\end{cases}
$$

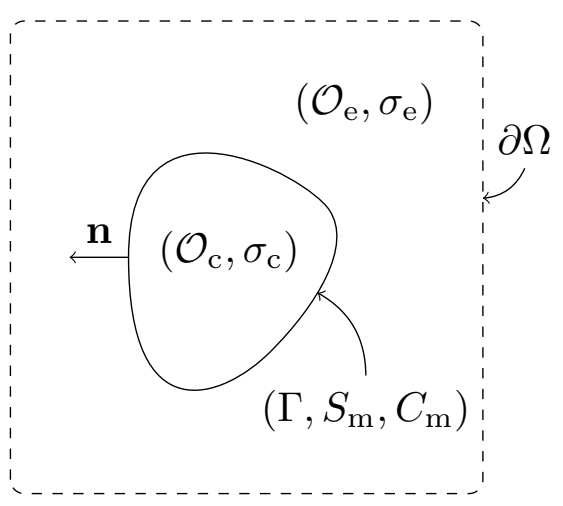

Figure 1: Geometry of the problem. The cell $\mathcal{O}_{\mathrm{c}}$ is imbedded in the bath $\mathcal{O}_{\mathrm{e}}$. The whole domain $\Omega$ is defined by $\Omega=\mathcal{O}_{\mathrm{e}} \cup \overline{\mathcal{O}_{\mathrm{c}}}$.

As described by Neu, Krassowska, and DeBruin [14, 3] the electric potential in the whole cell is the discontinuous solution $U$ to the following problem:

$$
\begin{aligned}
& \Delta U=0, \quad \text { in } \mathcal{O}_{\mathrm{e}} \cup \mathcal{O}_{\mathrm{c}}, \\
& U(0, \cdot)=0, \quad \text { and for all } t>0, \\
& U(t, x)=g(t, x), \quad \text { on } \partial \Omega,
\end{aligned}
$$

with the transmission conditions across the membrane $\Gamma$ :

$$
\begin{aligned}
& {\left[\sigma \partial_{\mathbf{n}} U\right]_{\Gamma}=0} \\
& C_{\mathrm{m}} \partial_{t}[U]_{\Gamma}+S_{\mathrm{m}}[U]_{\Gamma}=\sigma_{\mathrm{c}} \partial_{\mathbf{n}} U_{\left.\right|_{\Gamma^{-}}} .
\end{aligned}
$$

Throughout, the paper we denote by $\Omega$ the Lipschitz domain

$$
\Omega=\mathcal{O}_{\mathrm{e}} \cup \overline{\mathcal{O}_{\mathrm{c}}}
$$

and $\partial \Omega$ denotes its boundary. Moreover we define $P H^{1}(\Omega)$ as

$$
P H^{1}(\Omega)=\left\{u \in L^{2}(\Omega):\left.u\right|_{\mathcal{O}_{\mathrm{e}}} \in H^{1}\left(\mathcal{O}_{\mathrm{e}}\right),\left.u\right|_{\mathcal{O}_{\mathrm{c}}} \in H^{1}\left(\mathcal{O}_{\mathrm{c}}\right)\right\},
$$

and

$$
P H_{0}^{1}(\Omega)=\left\{u \in P H^{1}(\Omega):\left.u\right|_{\partial \Omega}=0\right\}
$$

\subsection{Electropermeabilization phenomenon}

When submitted to a high electric pulse - i.e. if the magnitude of the pulse $g$ reaches a threshold value - the cell membrane permeability increases and large molecules that usually cannot diffuse through the plasma membrane (for instance, plasmids or bleomycin) enter inside the cytoplasm. 
This phenomenon is called electroporation or electropermeabilization. For several years, different membrane models based on hydrodynamic, elasticity, hydroelasticity, viscoelasticity, or aqueous pore formation have been developed to describe the pore formation on the cell membrane (for more details, see the review of Pavlin et al. [17]). They all highlight a threshold value of the electric potential above which the electropermeabilization phenomenon occurs. However, the critical potential value changes with the models. Theoretical biophysicists consider the aqueous pore formation model as the most convincing current explanation. Nevertheless, the predictions of the model do not coincide with experiments either quantitatively or phenomenologically since no pore has ever been observed. In addition, the models based on the paper of Neu, Krassowska, et al. 14, 3, 4, are too complex to be parameterized to fit the experiments. Roughly speaking, the current models provide a qualitative explanation of the electropermeabilization, but the problem of the quantitative description remains open.

Actually in vitro and in vivo experiments have never proved the electropore formation, which theoretically could reach detectable size (since macropores could be created according to Smith et al. [21]); and it seems unclear whether electroporation results from holes punched in a lipid bilayer, as proposed in the current models (Teissié et al. 223, 22]). Moreover, the experimentally proved reversible process of the membrane electroporation is not clearly explained by the current models. In addition, and this is probably one of the main features of the experiments, the vectorization of large molecules requires both short time high-voltage pulses and long time low-voltage pulses (André et al. [1]). Therefore, the presence of pores is still controversial despite structural changes of the membrane. For all these reasons, we prefer the term electropermeabilization to electroporation.

\subsection{Modeling principle}

The probably most achieved electropermeabilization model has been proposed by Neu and Krassowska [14] and precisely described by DeBruin and Krassowska [3, 4]. It consists in adding an electroporation current $I_{\mathrm{ep}}$ in the right-hand side of equation (1e):

$$
C_{\mathrm{m}} \partial_{t}[U]_{\Gamma}+S_{\mathrm{m}}[U]_{\Gamma}=\sigma_{\mathrm{c}} \partial_{\mathbf{n}} U_{\Gamma_{\Gamma-}}+I_{\mathrm{ep}} .
$$

The current $I_{\mathrm{ep}}$ is given by a highly non-linear pore current $i_{\mathrm{ep}}$ multiplied by the pore density $N_{\text {ep }}$. The main drawback of such a model is its complexity since several parameters, such as the pore radius or the relative entrance length of the pore, which cannot be measured. Moreover, the mathematical well-posedness of the equations cannot be clearly established. Therefore, the inverse problem, that consists in fitting the parameters for each cell species, is hardly unsolvable numerically. In addition, the "philosophy" of the modeling is based on the pore creation, while as hinted above the very existence of these pores is controversial. For all these reasons, we choose to present here a new phenomenological model of electropermeabilization that could be fitted with the experiments. This model describes the membrane resealing and the memory of the applied pulses.

Our electropermeabilization modeling consists in describing the membrane permeabilization by choosing an appropriate function for the surface conductivity $S_{\mathrm{m}}$, instead of adding an electroporation current based on the pore creation as Neu, Krassowska, et al. did (see [14, 3, 21, 15]). In addition, we propose two models: the first one is a "static" model that describes the electropermeabilization as being an instantaneous phenomenon for a single time-constant pulse. This can be seen as a preliminary model that describes the cell potential during the pulse. The second model is the time-dependent model of electropermeabilization. For each model, we present the theoretical results that ensure existence and uniqueness of the solution to the new problems 
and then we present the numerical methods that allow the computation of the equations. We conclude by comparing our model with the model of Neu, Krassowska, et al.

\section{The static equation}

Based on the extensive review of Ivorra et al [10, the surface conductivity $S_{\mathrm{m}}$ is a function of the absolute membrane voltage, which tends to the value $S_{\mathrm{L}}$ (the lipid surface conductivity) below a certain threshold $V_{\text {rev }}$ (the reversible electropermeabilization voltage) and tends to $S_{\text {ir }}$ (the surface conductivity of the irreversibly electropermeabilized region) above this threshold, with $S_{\text {ir }}$ being larger than $S_{\mathrm{L}}$. The "speed of the switch" between these two values is given by a parameter $k_{\mathrm{ep}}$. We may choose the following sigmoid function for $S_{\mathrm{m}}$ :

$$
\forall \lambda \in \mathbb{R}, \quad S_{\mathrm{m}}(\lambda)=S_{\mathrm{L}}+\left(S_{\mathrm{ir}}-S_{\mathrm{L}}\right)\left[1+\tanh \left(k_{\mathrm{ep}}\left(|\lambda|-V_{\mathrm{rev}}\right)\right)\right] / 2,
$$

however other functions with similar monotonicity properties can be considered. More precisely, in our model the function $S_{\mathrm{m}}$ will satisfy the following condition:

$$
\left.\begin{array}{l}
S_{\mathrm{m}} \in C(\mathbb{R}), \lambda \mapsto S_{\mathrm{m}}(\lambda) \text { is even on } \mathbb{R}, \\
0<S_{\mathrm{L}} \leq S_{\mathrm{m}}(\lambda) \leq S_{\mathrm{ir}}, \quad S_{\mathrm{m}} \text { is non decreasing on }[0,+\infty), \\
\lim _{\lambda \rightarrow+\infty} S_{\mathrm{m}}(\lambda)=S_{\mathrm{ir}} .
\end{array}\right\}
$$

In particular, note that the mapping $\lambda \mapsto \lambda S_{\mathrm{m}}(\lambda)$ is increasing on $\mathbb{R}$.

Therefore, the static potential $U$ satisfies the following problem:

$$
\begin{aligned}
& \Delta U=0, \quad \text { in } \mathcal{O}_{\mathrm{e}} \cup \mathcal{O}_{\mathrm{c}}, \\
& {\left[\sigma \partial_{\mathbf{n}} U\right]_{\Gamma}=0, \quad \text { on } \Gamma,} \\
& S_{\mathrm{m}}\left([U]_{\Gamma}\right)[U]_{\Gamma}=\sigma_{\mathrm{c}} \partial_{\mathbf{n}} U_{\left.\right|_{\Gamma^{-}}}, \quad \text { on } \Gamma, \\
& U=g \quad \text { on } \partial \Omega .
\end{aligned}
$$

Remark 2.1. Model (4) can be seen as the limit of the model of Ivorra et al. [10], when the membrane thickness tends to zero (we refer to [18] for asymptotic expansion of the voltage potential in high contrast medium with resistive thin layer).

In the following subsections, we study the non-linear problem (4). In particular, we emphasize that due to the non-linearity of the membrane conductivity, increasing numerically the thickness of the membrane (as performed in [10]) leads to irrelevant results from the quantitative point of view. Therefore, we aim at providing efficient numerical methods in order to solve the above problem.

\subsection{Existence and uniqueness of the static potential}

This subsection is devoted to the proof of the following result.

Theorem 2.2. Let $g \in H^{1 / 2}(\partial \Omega)$. There exists a unique $U$ satisfying problem (4). This solution satisfies

$$
U_{\mathcal{O}_{\mathrm{e}}} \in H^{1}\left(\mathcal{O}_{\mathrm{e}}\right), \quad U_{\mathrm{O}_{\mathrm{c}}} \in H^{2}\left(\mathcal{O}_{\mathrm{c}}\right)
$$

In order to prove this theorem, we proceed as follows. Denote by $\Lambda_{\mathrm{c}}$ and $\Lambda_{\mathrm{e}}$ the Dirichlet-toNeumann operators on $\Gamma$ (also called Steklov-Poincaré operators) for the Laplacian respectively 
in $\mathcal{O}_{\mathrm{c}}$ and in $\mathcal{O}_{\mathrm{e}}$. More precisely, denote by $\mathbf{n}_{\mathrm{c}}$ (resp. $\left.\mathbf{n}_{\mathrm{e}}\right)$ the unitary outward normal to $\Gamma$ directed from the inside to the outside of $\mathcal{O}_{\mathrm{c}}\left(\right.$ resp. $\left.\mathcal{O}_{\mathrm{e}}\right)$. We define the operators $\Lambda_{\mathrm{c}}$ and $\Lambda_{\mathrm{e}}$ from $H^{1 / 2}(\Gamma)$ to $H^{-1 / 2}(\Gamma)$ as:

$$
\begin{aligned}
& \forall f \in H^{1 / 2}(\Gamma), \quad \Lambda_{\mathrm{c}}(f):=\mathbf{n}_{\mathrm{c}} \cdot \sigma_{\mathrm{c}} \nabla v_{\mathrm{c}_{\left.\right|_{\Gamma^{-}}}}, \text {where } \operatorname{div}\left(\sigma_{\mathrm{c}} \nabla v_{\mathrm{c}}\right)=0 \text { in } \mathcal{O}_{\mathrm{c}} \text {, and } v_{\mathrm{c}_{\left.\right|_{\Gamma}}}=f, \\
& \Lambda_{\mathrm{e}}(f):=\mathbf{n}_{\mathrm{e}} \cdot \sigma_{\mathrm{e}} \nabla v_{\mathrm{e}_{\Gamma^{+}}} \text {, where } \\
& \operatorname{div}\left(\sigma_{\mathrm{e}} \nabla v_{\mathrm{e}}\right)=0 \text { in } \mathcal{O}_{\mathrm{e}}, v_{\mathrm{e}_{\left.\right|_{\partial \Omega}}}=0 \text { and } v_{\mathrm{e}_{\mid}}=f .
\end{aligned}
$$

Observe that using Wirtinger-Poincaré's inequality in the case of $\Lambda_{\mathrm{c}}$, or Poincaré's inequality in the case of $\Lambda_{\mathrm{e}}$, together with the continuity of the mapping $u \mapsto u_{\mid \partial \mathcal{O}}$ from $H^{1}(\mathcal{O})$ into $H^{1 / 2}(\partial \mathcal{O})$, when $\mathcal{O}$ is sufficiently smooth, the following inequalities hold:

$$
\begin{aligned}
& \left\langle\Lambda_{\mathrm{c}} f, f\right\rangle=\int_{\mathcal{O}_{\mathrm{c}}} \sigma_{\mathrm{c}}(x) \nabla v(x) \cdot \nabla v(x) \mathrm{d} x \geq C_{\mathrm{c}}\|f-M(f)\|_{H^{1 / 2}(\Gamma)}^{2}, \\
& \left\langle\Lambda_{\mathrm{e}} f, f\right\rangle=\int_{\mathcal{O}_{\mathrm{e}}} \sigma_{\mathrm{e}}(x) \nabla v_{\mathrm{e}}(x) \cdot \nabla v_{\mathrm{e}}(x) \mathrm{d} x \geq C_{\mathrm{e}}\|f\|_{H^{1 / 2}(\Gamma)}^{2},
\end{aligned}
$$

where $M(f)=|\Gamma|^{-1} \int_{\Gamma} f(\tau) \mathrm{d} \tau$ is the mean value of $f$ on $\Gamma$, and $C_{\mathrm{e}}$ and $C_{\mathrm{c}}$ are constants depending only on $\mathcal{O}_{\mathrm{e}}$ and $\mathcal{O}_{\mathrm{c}}$ respectively. Moreover, for a function $g \in H^{1 / 2}(\partial \Omega)$, we define $\Lambda_{0}(g)$ by:

$$
\begin{aligned}
\Lambda_{0}(g):=\mathbf{n}_{\mathrm{e}} \cdot \sigma_{\mathrm{e}} \nabla v_{\left.\right|_{\Gamma^{+}}}, \quad \text { where } \\
\qquad \operatorname{div}\left(\sigma_{\mathrm{e}} \nabla v\right)=0 \text { in } \mathcal{O}_{\mathrm{e}}, \quad v_{\left.\right|_{\partial \Omega}}=g \text { and } v_{\left.\right|_{\Gamma}}=0 .
\end{aligned}
$$

It is useful to recall that the operator $\Lambda_{\mathrm{e}}$ is invertible, its inverse being given by another SteklovPoincaré operator (or what is sometimes called a Neumann-to-Dirichlet operator). Namely, for any $\psi \in H^{-1 / 2}(\Gamma)$ given, one has $\Lambda_{\mathrm{e}}^{-1}(\psi)=v_{\mid \Gamma}$ where $v \in H^{1}\left(\mathcal{O}_{\mathrm{e}}\right)$ satisfies the equation

$$
\operatorname{div}\left(\sigma_{\mathrm{e}} \nabla v\right)=0 \text { in } \mathcal{O}_{\mathrm{e}}, v_{\mid \partial \Omega}=0 \text { and } \mathbf{n}_{\mathrm{e}} \cdot \sigma_{\mathrm{e}} \nabla v_{\mid \Gamma^{+}}=\psi
$$

Consider the Hilbert space $\mathbb{H}$ defined by

$$
\mathbb{H}=H^{1 / 2}(\Gamma) \times H^{1 / 2}(\Gamma),
$$

with the norm

$$
\forall \mathbf{u}=\left(u_{\mathrm{e}}, u_{\mathrm{c}}\right) \in \mathbb{H}, \quad\|\mathbf{u}\|_{\mathbb{H}}^{2}=\left\|u_{\mathrm{e}}\right\|_{H^{1 / 2}(\Gamma)}^{2}+\left\|u_{\mathrm{c}}\right\|_{H^{1 / 2}(\Gamma)}^{2} .
$$

Problem (4) can be written on the manifold $\Gamma$ with the help of the above Steklov-Poincaré operators. More precisely, problem (4) is equivalent to finding $\left(u_{\mathrm{e}}, u_{\mathrm{c}}\right) \in \mathbb{H}$ such that

$$
\begin{aligned}
& \Lambda_{\mathrm{e}} u_{\mathrm{e}}+S_{\mathrm{m}}\left(u_{\mathrm{e}}-u_{\mathrm{c}}\right)\left(u_{\mathrm{e}}-u_{\mathrm{c}}\right)=-\Lambda_{0}(g), \\
& \Lambda_{\mathrm{c}} u_{\mathrm{c}}-S_{\mathrm{m}}\left(u_{\mathrm{e}}-u_{\mathrm{c}}\right)\left(u_{\mathrm{e}}-u_{\mathrm{c}}\right)=0 .
\end{aligned}
$$

Notation 2.3. Identifying the dual of $L^{2}(\Gamma) \times L^{2}(\Gamma)$ with $L^{2}(\Gamma) \times L^{2}(\Gamma)$, we denote by $\mathbb{H}^{\prime}$ the dual space of $\mathbb{H}$ and by $\langle\cdot, \cdot\rangle$ the duality between $\mathbb{H}$ and $\mathbb{H}^{\prime}$.

The proof of Theorem 2.2 is an obvious application of the following theorem.

Theorem 2.4. Let $\mathbf{G}=\left(G_{\mathrm{e}}, G_{\mathrm{c}}\right) \in \mathbb{H}^{\prime}$. There exists a unique $\mathbf{u}^{\mathbf{0}}=\left(u_{\mathrm{e}}, u_{\mathrm{c}}\right) \in \mathbb{H}$ such that

$$
\begin{aligned}
& \Lambda_{\mathrm{e}} u_{\mathrm{e}}+S_{\mathrm{m}}\left(u_{\mathrm{e}}-u_{\mathrm{c}}\right)\left(u_{\mathrm{e}}-u_{\mathrm{c}}\right)=G_{\mathrm{e}} \\
& \Lambda_{\mathrm{c}} u_{\mathrm{c}}-S_{\mathrm{m}}\left(u_{\mathrm{e}}-u_{\mathrm{c}}\right)\left(u_{\mathrm{e}}-u_{\mathrm{c}}\right)=G_{\mathrm{c}} .
\end{aligned}
$$


Proof. We define the operator $\boldsymbol{\Lambda}_{\sigma}$ from $\mathbb{H}$ into $\mathbb{H}^{\prime}$ by

$$
\forall \mathbf{u} \in \mathbb{H}, \quad \boldsymbol{\Lambda}_{\sigma} \mathbf{u}=\left(\begin{array}{c}
\Lambda_{\mathrm{e}} u_{\mathrm{e}} \\
\Lambda_{\mathrm{c}} u_{\mathrm{c}}
\end{array}\right)=\left(\begin{array}{cc}
\Lambda_{\mathrm{e}} & 0 \\
0 & \Lambda_{\mathrm{c}}
\end{array}\right)\left(\begin{array}{l}
u_{\mathrm{e}} \\
u_{\mathrm{c}}
\end{array}\right) .
$$

Thanks to (6) and (7), we have

$$
\forall \mathbf{u} \in \mathbb{H}, \quad\left\langle\boldsymbol{\Lambda}_{\sigma} \mathbf{u}, \mathbf{u}\right\rangle \geq C_{\mathrm{e}}\left\|u_{\mathrm{e}}\right\|_{H^{1 / 2}(\Gamma)}^{2}+C_{\mathrm{c}}\left\|u_{\mathrm{c}}-M\left(u_{\mathrm{c}}\right)\right\|_{H^{1 / 2}(\Gamma)}^{2} .
$$

Since the function $S_{\mathrm{m}}$ satisfies conditions (3), we introduce the function $F$ defined by

$$
\forall s \in \mathbb{R}, \quad F(s)=\int_{0}^{s} S_{\mathrm{m}}(z) z \mathrm{~d} z .
$$

Note that $F$ is even, that is $F(-s)=F(s)$. Let $\mathbf{J}_{\mathbf{1}}$ be the function defined on $\mathbb{H}$ by

$$
\forall \mathbf{u} \in \mathbb{H}, \quad \mathbf{J}_{\mathbf{1}}(\mathbf{u})=\mathbf{J}_{\mathbf{1}}\left(u_{\mathrm{e}}, u_{\mathrm{c}}\right)=\int_{\Gamma} F\left(u_{\mathrm{e}}(\tau)-u_{\mathrm{c}}(\tau)\right) \mathrm{d} \tau
$$

One easily checks that $\mathbf{J}_{\mathbf{1}}$ is a $C^{1}$ function on $\mathbb{H}$ and

$$
\mathbf{J}_{\mathbf{1}}(\mathbf{u}) \geq \frac{1}{2} S_{\mathrm{L}} \int_{\Gamma}\left|u_{\mathrm{e}}(\tau)-u_{\mathrm{c}}(\tau)\right|^{2} \mathrm{~d} \tau
$$

Observe that, for any $\mathbf{u} \in \mathbb{H}$, the derivative $\mathbf{J}_{\mathbf{1}}^{\prime}(\mathbf{u})$ of $\mathbf{J}_{\mathbf{1}}$ at $\mathbf{u}$ is the linear map defined by

$$
\forall \mathbf{h} \in \mathbb{H}, \quad \mathbf{J}_{\mathbf{1}}^{\prime}(\mathbf{u}) \cdot \mathbf{h}=\int_{\Gamma} S_{m}\left(u_{\mathrm{e}}(\tau)-u_{\mathrm{c}}(\tau)\right)\left(u_{\mathrm{e}}(\tau)-u_{\mathrm{c}}(\tau)\right)\left(h_{\mathrm{e}}(\tau)-h_{\mathrm{c}}(\tau)\right) \mathrm{d} \tau
$$

Let $\mathbf{J}$ be defined by

$$
\forall \mathbf{u} \in \mathbb{H}, \quad \mathbf{J}(\mathbf{u})=\frac{1}{2}\left\langle\boldsymbol{\Lambda}_{\sigma} \mathbf{u}, \mathbf{u}\right\rangle+\mathbf{J}_{\mathbf{1}}(\mathbf{u})-\langle\mathbf{G}, \mathbf{u}\rangle .
$$

$\mathbf{J}$ is of class $\mathscr{C}^{1}$ on $\mathbb{H}$ and $\mathbf{J}^{\prime}$ is given by

$$
\forall \mathbf{u} \in \mathbb{H}, \quad \mathbf{J}^{\prime}(\mathbf{u})=\left(\begin{array}{c}
\Lambda_{\mathrm{e}} u_{\mathrm{e}}+S_{\mathrm{m}}\left(u_{\mathrm{e}}-u_{\mathrm{c}}\right)\left(u_{\mathrm{e}}-u_{\mathrm{c}}\right)-G_{\mathrm{e}} \\
\Lambda_{\mathrm{c}} u_{\mathrm{c}}-S_{\mathrm{m}}\left(u_{\mathrm{e}}-u_{\mathrm{c}}\right)\left(u_{\mathrm{e}}-u_{\mathrm{c}}\right)-G_{\mathrm{c}}
\end{array}\right)
$$

In order to show that $\mathbf{J}^{\prime}$ is a monotone operator, we define the nonlinear operator $B$ from $\mathbb{H}^{2}$ into $\mathbb{R}$ by

$$
\forall(\mathbf{u}, \mathbf{v}) \in \mathbb{H}^{2}, \quad B(\mathbf{u}, \mathbf{v})=S_{\mathrm{m}}([\mathbf{u}])[\mathbf{u}]^{2}\left(1-\frac{[\mathbf{v}]}{[\mathbf{u}]}\right)\left(1-\frac{S_{\mathrm{m}}([\mathbf{v}])}{S_{\mathrm{m}}([\mathbf{u}])} \frac{[\mathbf{v}]}{[\mathbf{u}]}\right)
$$

where for simplicity we denote by $[\mathbf{u}]=u_{\mathrm{e}}-u_{\mathrm{c}}$ for $\mathbf{u}=\left(u_{\mathrm{e}}, u_{\mathrm{c}}\right) \in \mathbb{H}$. Taking into account the fact that $S_{\mathrm{m}}$ satisfies (3), one checks easily that $B(\mathbf{u}, \mathbf{v}) \geq 0$. According to $(12)$ and (13), for $(\mathbf{u}, \mathbf{v}) \in \mathbb{H}^{2}$ we have

$$
\left\langle\mathbf{J}^{\prime}(\mathbf{u})-\mathbf{J}^{\prime}(\mathbf{v}), \mathbf{u}-\mathbf{v}\right\rangle=\left\langle\boldsymbol{\Lambda}_{\sigma}(\mathbf{u}-\mathbf{v}), \mathbf{u}-\mathbf{v}\right\rangle+\int_{\Gamma} B(\mathbf{u}(s), \mathbf{v}(s)) \mathrm{d} s
$$

from which we infer that $\mathbf{J}^{\prime}$ is a monotone operator. Therefore $\mathbf{J}$ is convex. In order to see the strict convexity of $\mathbf{J}$, that is the strict monotonicity of $\mathbf{J}^{\prime}$, we have to show that if for a given

$\operatorname{RR} \mathrm{n}^{\circ} 8005$ 
$\mathbf{u}, \mathbf{v} \in \mathbb{H}$ we have $\left\langle\mathbf{J}^{\prime}(\mathbf{u})-\mathbf{J}^{\prime}(\mathbf{v}), \mathbf{u}-\mathbf{v}\right\rangle=0$, then we have $\mathbf{u}=\mathbf{v}$. Observe first that, since $\lambda \mapsto \lambda S_{\mathrm{m}}(\lambda)$ is increasing, we have that

$$
B(\mathbf{u}, \mathbf{v})=0 \quad \Longrightarrow \quad[\mathbf{u}]=[\mathbf{v}] .
$$

In particular, for these $\mathbf{u}, \mathbf{v}$, we have $B(\mathbf{u}, \mathbf{v})=0$, which implies $[\mathbf{u}]=[\mathbf{v}]$, hence $u_{\mathrm{e}}-v_{\mathrm{e}}=u_{\mathrm{c}}-v_{\mathrm{c}}$. On the other hand, since

$$
\left\langle\Lambda_{\mathrm{e}}\left(u_{\mathrm{e}}-v_{\mathrm{e}}\right), u_{\mathrm{e}}-v_{\mathrm{e}}\right\rangle=\left\langle\Lambda_{\mathrm{c}}\left(u_{\mathrm{c}}-v_{\mathrm{c}}\right), u_{\mathrm{c}}-v_{\mathrm{c}}\right\rangle=0
$$

and since $\Lambda_{\mathrm{e}}$ is coercive, we conclude that $u_{\mathrm{e}}-v_{\mathrm{e}}=0$, which in turn implies that $u_{\mathrm{c}}-v_{\mathrm{c}}=0$. Finally, this shows that

$$
\left\langle\mathbf{J}^{\prime}(\mathbf{u})-\mathbf{J}^{\prime}(\mathbf{v}), \mathbf{u}-\mathbf{v}\right\rangle=0 \quad \Longrightarrow \quad \mathbf{u}=\mathbf{v}
$$

Therefore, $\mathbf{J}^{\prime}$ is strictly monotone and $\mathbf{J}$ is strictly convex.

In order to show the coerciveness of $\mathbf{J}$, that is $\mathbf{J}(\mathbf{u}) \rightarrow+\infty$ when $\|\mathbf{u}\|_{\mathbb{H}} \rightarrow \infty$, we proceed as follows. Observe first that

$$
\begin{gathered}
\left|\left\langle G_{\mathrm{c}}, M\left(u_{\mathrm{c}}\right)\right\rangle\right| \leq\left\|G_{\mathrm{c}}\right\|_{H^{-1 / 2}(\Gamma)}\left\|M\left(u_{\mathrm{e}}-u_{\mathrm{c}}\right)\right\|_{H^{1 / 2}(\Gamma)} \\
+\left\|G_{\mathrm{c}}\right\|_{H^{-1 / 2}(\Gamma)}\left\|M\left(u_{\mathrm{e}}\right)\right\|_{H^{1 / 2}(\Gamma)}, \\
\leq\left\|G_{\mathrm{C}}\right\|_{H^{-1 / 2}(\Gamma)}\left\|M\left(u_{\mathrm{e}}-u_{\mathrm{c}}\right)\right\|_{H^{1 / 2}(\Gamma)} \\
+\left\|G_{\mathrm{c}}\right\|_{H^{-1 / 2}(\Gamma)}\left\|u_{\mathrm{e}}\right\|_{H^{1 / 2}(\Gamma)},
\end{gathered}
$$

hence

$$
\begin{aligned}
|\langle\mathbf{G}, \mathbf{u}\rangle| \leq & \left(\left\|G_{\mathrm{e}}\right\|_{H^{-1 / 2}(\Gamma)}+\left\|G_{\mathrm{c}}\right\|_{H^{-1 / 2}(\Gamma)}\right)\left\|u_{\mathrm{e}}\right\|_{H^{1 / 2}(\Gamma)} \\
& +\left\|G_{\mathrm{c}}\right\|_{H^{-1 / 2}(\Gamma)}\left\|u_{\mathrm{c}}-M\left(u_{\mathrm{c}}\right)\right\|_{H^{1 / 2}(\Gamma)} \\
& +\left\|G_{\mathrm{c}}\right\|_{H^{-1 / 2}(\Gamma)}\left\|M\left(u_{\mathrm{e}}-u_{\mathrm{c}}\right)\right\|_{H^{1 / 2}(\Gamma)} .
\end{aligned}
$$

Using Young's inequality $\left(a b \leq \varepsilon a^{2}+C(\varepsilon) b^{2}\right.$, for $\varepsilon>0$ and $\left.C(\varepsilon):=(4 \varepsilon)^{-1}\right)$ we infer

$$
\begin{aligned}
|\langle\mathbf{G}, \mathbf{u}\rangle| \leq & \varepsilon\left\|u_{\mathrm{e}}\right\|_{H^{1 / 2}(\Gamma)}^{2}+\varepsilon\left\|u_{\mathrm{c}}-M\left(u_{\mathrm{c}}\right)\right\|_{H^{1 / 2}(\Gamma)}^{2}+2 C(\varepsilon)\|\mathbf{G}\|_{\mathbb{H}^{\prime}}^{2} \\
& +\left\|G_{\mathrm{c}}\right\|_{H^{-1 / 2}(\Gamma)}\left\|M\left(u_{\mathrm{e}}-u_{\mathrm{c}}\right)\right\|_{H^{1 / 2}(\Gamma)} .
\end{aligned}
$$

On the other hand, using the fact that $2 F(s) \geq S_{\mathrm{L}} s^{2}$ for $s \in \mathbb{R}$, we deduce that

$$
\begin{aligned}
\mathbf{J}_{\mathbf{1}}(\mathbf{u}) & \geq \frac{1}{2} S_{\mathrm{L}} \int_{\Gamma}\left|u_{\mathrm{e}}(\tau)-u_{\mathrm{c}}(\tau)\right|^{2} \mathrm{~d} \tau \\
& \geq \frac{1}{2} S_{\mathrm{L}}\left(\int_{\Gamma}\left|\left(u_{\mathrm{e}}-u_{\mathrm{c}}\right)-M\left(u_{\mathrm{e}}-u_{\mathrm{c}}\right)\right|^{2} \mathrm{~d} \tau+\int_{\Gamma}\left|M\left(u_{\mathrm{e}}-u_{\mathrm{c}}\right)\right|^{2} \mathrm{~d} \tau\right) .
\end{aligned}
$$

Using this inequality, together with $(16)$, we obtain a lower bound for $\mathbf{J}(\mathbf{u})$ (here $a(\varepsilon), C(\varepsilon)$ are positive constants depending on the arbitrary $\varepsilon>0$, and $b>0$ is a constant):

$$
\begin{aligned}
\mathbf{J}(\mathbf{u}) \geq & a(\varepsilon)\left(\left\|u_{\mathrm{e}}\right\|_{H^{1 / 2}(\Gamma)}^{2}+\left\|u_{\mathrm{c}}-M\left(u_{\mathrm{c}}\right)\right\|_{H^{1 / 2}(\Gamma)}^{2}\right) \\
& +b \int_{\Gamma}\left|M\left(u_{\mathrm{e}}-u_{\mathrm{c}}\right)\right|^{2} \mathrm{~d} \tau-\left\|G_{\mathrm{c}}\right\|_{H^{-1 / 2}(\Gamma)}\left\|M\left(u_{\mathrm{e}}-u_{\mathrm{c}}\right)\right\|_{H^{1 / 2}(\Gamma)} \\
& \quad-C(\varepsilon)\|\mathbf{G}\|_{\mathbb{H}^{\prime}}^{2} .
\end{aligned}
$$


Since $M\left(u_{\mathrm{e}}-u_{\mathrm{c}}\right)$ is a constant, we observe that one has $\left\|M\left(u_{\mathrm{e}}-u_{\mathrm{c}}\right)\right\|_{H^{1 / 2}(\Gamma)}=c_{*} \| M\left(u_{\mathrm{e}}-\right.$ $\left.u_{\mathrm{c}}\right) \|_{L^{2}(\Gamma)}$ for some positive constant $c_{*}$ independent of $\mathbf{u}$. Consequentely, for any $\varepsilon>0$ so that $b-\varepsilon>0$, there exists a constant $c(\varepsilon)$ such that

$$
\begin{aligned}
b \int_{\Gamma}\left|M\left(u_{\mathrm{e}}-u_{\mathrm{c}}\right)\right|^{2} \mathrm{~d} \tau-\left\|G_{\mathrm{c}}\right\|_{H^{-1 / 2}(\Gamma)}\left\|M\left(u_{\mathrm{e}}-u_{\mathrm{c}}\right)\right\|_{H^{1 / 2}(\Gamma)} \geq \\
(b-\varepsilon)\left\|M\left(u_{\mathrm{e}}-u_{\mathrm{c}}\right)\right\|_{H^{1 / 2}(\Gamma)}^{2}-c(\varepsilon)\left\|G_{\mathrm{c}}\right\|_{H^{-1 / 2}(\Gamma)}^{2} .
\end{aligned}
$$

Using this inequality in the lower bound 17 , we conclude that

$$
\lim _{\|\mathbf{u}\|_{\mathbb{H}} \rightarrow+\infty} \mathbf{J}(\mathbf{u})=+\infty
$$

hence $\mathbf{J}$ achieves its minimum at a unique point $\mathbf{u}^{\mathbf{0}} \in \mathbb{H}$, which satisfies equation (10).

\section{The dynamical model}

In this section, we focus on the dynamical description of the electropermeabilization. Our model is based on the description of two quantities: the time-dependent electric potential and the ratio of the electropermeabilized region over the total membrane area, which is also time-dependent. In subsection 3.1, we present the main considerations that lead to our model. We then study its solvability: existence and uniqueness results are presented in subsection 3.4

\subsection{Heuristics of the modeling}

Experimental observations suggest that the permeabilization process at a certain location depends on whether the membrane conductivity is above a certain threshold or not. This leads us to define the surface membrane conductivity as an interpolation between the two values $S_{\text {ir }}$ and $S_{\mathrm{L}}$, the interpolation parameter $\xi(t, s) \in[0,1]$ being itself a function of time and of the point $s$ on the membrane $\Gamma$. In our interpretation, the parameter $\xi(t, s)$ measures in some way the likelihood that a given infinitesimal portion of the membrane is going to be electropermeabilized. More precisely, when $\xi(t, s)$ equals 0 at a given point $s \in \Gamma$, the membrane conductivity equals the lipid conductivity at this point (thus there is no electropermeabilization), while for $\xi(t, s)=1$ it corresponds to the maximal value of the membrane surface conductivity above which electropermeabilization is irreversible. Thus the time-dependent membrane conductivity, denoted by $S_{\mathrm{m}}$ writes

$$
\forall(t, s) \in(0,+\infty) \times \Gamma, \quad S_{\mathrm{m}}(t, s)=S_{\mathrm{L}}+\xi(t, s)\left(S_{\mathrm{ir}}-S_{\mathrm{L}}\right) .
$$

On the other hand, the changes in the conductivity at a certain location $s \in \Gamma$ depend on the transmembrane voltage. Denoting by $[\mathbf{u}]:=u_{\mathrm{e}}-u_{\mathrm{c}}$ the jump in the potential between the outside and the inside of the cell for $\mathbf{u}:=\left(u_{\mathrm{e}}, u_{\mathrm{c}}\right) \in \mathbb{H}$ (as we did in the previous sections), we therefore assume that

$$
\xi(t, s)=X(t,[\mathbf{u}(t, s)]),
$$

where the function $(t, \lambda) \mapsto X(t, \lambda)$ will be defined below.

The main idea of the modeling consists in writing a differential equation that describes the dynamics of $(t, \lambda) \mapsto X(t, \lambda)$ similarly to a sliding door model. Let $\beta$ be a function satisfying

$R R n^{\circ} 8005$

$$
\begin{aligned}
& \beta \in W^{1, \infty}(\mathbb{R}), \lambda \mapsto \beta(\lambda) \text { is even on } \mathbb{R}, \\
& \lambda \mapsto \lambda \beta^{\prime}(\lambda) \quad \text { belongs to } L^{\infty}(\mathbb{R}), \\
& 0 \leq \beta(\lambda) \leq 1, \quad \beta \text { is non decreasing on }(0,+\infty), \\
& \lim _{\lambda \rightarrow+\infty} \beta(\lambda)=1 .
\end{aligned}
$$


An example of such a function would be

$$
\forall \lambda \in \mathbb{R}, \quad \beta(\lambda):=\left(1+\tanh \left(k_{\mathrm{ep}}\left(|\lambda|-V_{\text {rev }}\right)\right) / 2 .\right.
$$

Let us describe now the evolution of $X$. For $\lambda_{0} \in \mathbb{R}$, which will stand for a given $\Delta \mathrm{TMP}$, and for an initial value $X_{0} \in[0,1]$ of $\mathrm{X}$, set $\beta_{0}:=\beta\left(\lambda_{0}\right)$. Then we consider the following two possibilities:

- Either $\beta_{0}-X_{0}$ is positive, in which case the electric pulse is sufficiently high to enlarge the electropermeabilized region, with a characteristic time of electropermeabilization of order $\tau_{\text {ep }}$.

- Or $\beta_{0}-X_{0}$ is negative, in which case we consider that the pulse is not high enough to increase the electropermeabilization. Therefore, the membrane tries to reseal with a characteristic resealing time of order $\tau_{\text {res }}$. Since experimental observations suggest that this phenomenon takes much more time than the electropermeabilization process, we assume that $\tau_{\text {res }}>\tau_{\text {ep }}$.

Remark that when a cell is at rest, $X_{0}$ equals zero, but if high voltage pulses have been applied earlier than the initial time, $X_{0}$ might not be equal to zero.

Based on these considerations, for any $\lambda_{0} \in \mathbb{R}$, we assume that $X\left(\cdot, \lambda_{0}\right)$ satisfies the following differential equation:

$$
\left\{\begin{array}{l}
\frac{\partial X}{\partial t}\left(t, \lambda_{0}\right)=\max \left(\frac{\beta\left(\lambda_{0}\right)-X\left(t, \lambda_{0}\right)}{\tau_{\mathrm{ep}}} ; \frac{\beta\left(\lambda_{0}\right)-X\left(t, \lambda_{0}\right)}{\tau_{\text {res }}}\right), \quad \forall t>0 \\
X\left(0, \lambda_{0}\right)=X_{0} .
\end{array}\right.
$$

\subsection{Statement of the mathematical problem}

We first write the equation satisfied by the potential $U$ defined on the domain $\mathcal{O}_{\mathrm{e}} \cup \mathcal{O}_{\mathrm{c}}$. We assume that before the imposition of the electrical pulses $g$ on the external boundary $\partial \Omega$, the cell potential is at rest and given by $U_{0} \in H^{1}(\Omega)$. This resting potential translates the ionic exchanges through the membrane. According to equalities $(18)-(19)$, the membrane conductivity $\widetilde{S}_{\mathrm{m}}$ is defined by:

$$
\widetilde{S}_{\mathrm{m}}(t, \lambda):=S_{\mathrm{L}}+\left(S_{\mathrm{ir}}-S_{\mathrm{L}}\right) X(t, \lambda)
$$

We seek the solution $(U, X)$ to the following system of equations: for $\left(U_{0}, X_{0}\right)$ given, the pair of functions $(U, X)$ satisfy:

$$
\begin{aligned}
\left.U\right|_{t=0}=U_{0}, \quad & \text { and for any } t>0, \\
& \Delta U=0, \quad \text { in }(0, T) \times\left(\mathcal{O}_{\mathrm{e}} \cup \mathcal{O}_{\mathrm{c}}\right), \quad U(t, \cdot)=g(t, \cdot) \quad \text { on }(0,+\infty) \times \partial \Omega, \\
& {\left[\sigma \partial_{\mathbf{n}} U\right]=0, \quad \text { on }(0, T) \times \Gamma, } \\
& C_{\mathrm{m}} \partial_{t}[U](t, \cdot)+\widetilde{S}_{\mathrm{m}}(t,[U])[U]=\sigma_{\mathrm{c}} \partial_{\mathbf{n}} U(t, \cdot)_{\Gamma^{-}}, \quad \text { on }(0, T) \times \Gamma,
\end{aligned}
$$

where, writing $\lambda=[U](t, s)$, the function $X$ appearing in 22 satisfies the differential equation

$$
\begin{aligned}
& \frac{\partial X(t, \lambda)}{\partial t}=\max \left(\frac{\beta(\lambda)-X(t, \lambda)}{\tau_{\mathrm{ep}}} ; \frac{\beta(\lambda)-X(t, \lambda)}{\tau_{\mathrm{res}}}\right), \quad t>0, \\
& X(0, \lambda)=X_{0} .
\end{aligned}
$$


Remark 3.1. Observe that if the source $g$ does not depend on the time $t$, the stationary point $\left(U^{*}, X^{*}\right)$ of the system $(23)-(24 \mathrm{a})$ is the unique solution to

$$
\begin{aligned}
& \Delta U^{*}=0, \quad \text { in }\left(\mathcal{O}_{\mathrm{e}} \cup \mathcal{O}_{\mathrm{c}}\right),\left.\quad U^{*}\right|_{\partial \Omega}=g \quad \text { on } \partial \Omega, \\
& {\left[\sigma \partial_{\mathbf{n}} U^{*}\right]=0, \quad \text { on } \Gamma,} \\
& \left(S_{\mathrm{L}}+\left(S_{\mathrm{ir}}-S_{\mathrm{L}}\right) X^{*}\right)\left[U^{*}\right]=\sigma_{\mathrm{c}} \partial_{\mathbf{n}} U_{\Gamma_{\Gamma^{-}}^{*}}, \quad \text { on } \Gamma,
\end{aligned}
$$

where

$$
X^{*}=\beta\left(\left[U^{*}(s)\right]\right),
$$

which coincides with the static model (2).

\subsection{Properties of the function $X$}

Let us state the following lemma regarding the solution to equation (24):

Lemma 3.2. Let $T>0$ be fixed and let $\beta$ satisfy condition 20 . For $T>0$ and any $\lambda \in \mathbb{R}$, the following differential equation

$$
\left\{\begin{array}{l}
\frac{\partial X(t, \lambda)}{\partial t}=\max \left(\frac{\beta(\lambda)-X(t, \lambda)}{\tau_{\mathrm{ep}}} ; \frac{\beta(\lambda)-X(t, \lambda)}{\tau_{\mathrm{res}}}\right), \quad \forall t \in(0, T), \\
X(0, \lambda)=X_{0} \in[0,1]
\end{array}\right.
$$

has a unique solution $X(\cdot, \lambda) \in C^{1}([0, T])$. Moreover one has

$$
0 \leq X(t, \lambda)=X(t,-\lambda)=X(t,|\lambda|) \leq 1, \quad \forall t \in[0, T], \quad \forall \lambda \in \mathbb{R} .
$$

In addition, there exists a constant $K(T)>0$ such that for any $\lambda_{1}, \lambda_{2} \in \mathbb{R}$ we have

$$
\forall t \in[0, T], \quad\left|\lambda_{1} X\left(t, \lambda_{1}\right)-\lambda_{2} X\left(t, \lambda_{2}\right)\right| \leq K(T)\left|\lambda_{1}-\lambda_{2}\right| .
$$

Proof. The mapping $X \mapsto \max \left([\beta(\lambda)-X] / \tau_{\text {ep }},[\beta(\lambda)-X] / \tau_{\text {res }}\right)$, defined from $\mathbb{R}$ into itself, is clearly Lipschitz for all fixed $\lambda$. Therefore, the differential equation (25) has a unique solution $X \in C^{1}([0, T])$, for any given $X_{0} \in \mathbb{R}$.

Assuming now that $0 \leq X_{0} \leq 1$, multiplying the equation 25 by $X^{-}:=\max (-X, 0)$, and using the fact that $\beta \geq 0$, one gets

$$
\frac{1}{2} \partial_{t}\left|X^{-}\right|^{2} \leq 0
$$

Thus $X^{-}(\cdot, \lambda) \equiv 0$ on $[0, T]$. Similarly, using the fact that $\beta-1 \leq 0$, and multiplying the equation by $(X-1)^{+}=\max (X-1,0)$, one sees that

$$
\partial_{t}\left|(X-1)^{+}\right|^{2} \leq 0
$$

and finally $0 \leq X(t, \lambda) \leq 1$, for any $t \in[0, T]$.

In order to show the estimate $(26)$, we proceed as follows. For $\lambda \in \mathbb{R}$ denote by $Y(t, \lambda):=$ $\lambda X(t, \lambda)$. Since for any $(a, b) \in \mathbb{R}$, we have $\max (a, b)=a+(a-b)^{-}$, assuming for instance that $\tau_{\text {ep }}<\tau_{\text {res }}$ we observe that

$$
\max \left(a / \tau_{\text {ep }}, a / \tau_{\text {res }}\right)=\frac{a}{\tau_{\text {ep }}}+\left(\tau_{\text {ep }}^{-1}-\tau_{\text {res }}^{-1}\right) a^{-},
$$

$\mathrm{RR} \mathrm{n}^{\circ} 8005$ 
and using the equality $a^{-}=(|a|-a) / 2$ we get

$$
\max \left(a / \tau_{\mathrm{ep}}, a / \tau_{\text {res }}\right)=a\left(\tau_{\mathrm{ep}}^{-1}+\tau_{\text {res }}^{-1}\right) / 2+|a|\left(\tau_{\mathrm{ep}}^{-1}-\tau_{\text {res }}^{-1}\right) / 2 .
$$

From this we induce that $Y$ satisfies the following O.D.E

$$
\left\{\begin{array}{l}
\frac{\partial Y(t, \cdot)}{\partial t}=H(\lambda, Y), \quad \forall t \in(0, T), \\
Y(0, \lambda)=Y_{0}=\lambda X_{0}
\end{array}\right.
$$

where the function $H$ is defined by

$$
H:(\lambda, Y) \mapsto H(\lambda, Y)=(\lambda \beta(\lambda)-Y)\left(\tau_{\text {ep }}^{-1}+\tau_{\text {res }}^{-1}\right) / 2+\lambda|\beta(\lambda)-Y / \lambda|\left(\tau_{\text {ep }}^{-1}-\tau_{\text {res }}^{-1}\right) / 2 .
$$

In order to see that the mapping $\lambda \mapsto H(\lambda, Y)$ is globally Lipschitz on $\mathbb{R}$, since by our assumptions on the function $\beta$ we know that $\lambda \beta^{\prime}(\lambda)$ is uniformly bounded on $\mathbb{R}$, the function $\lambda \mapsto \lambda \beta(\lambda)$ is Lipschitz on $\mathbb{R}$, and thus we have only to verify that the function $h: \lambda \mapsto h(\lambda, Y)=\lambda|\beta(\lambda)-Y / \lambda|$ is Lipschitz on $\mathbb{R}$. Indeed, this is the case as one can see by a simple computation that

$$
\begin{aligned}
\frac{\partial h}{\partial \lambda} & =|\beta(\lambda)-Y / \lambda|+\left(\lambda \beta^{\prime}(\lambda)+Y / \lambda\right) \frac{\beta(\lambda)-Y / \lambda}{|\beta(\lambda)-Y / \lambda|}, \\
& =\left(\beta(\lambda)+\lambda \beta^{\prime}(\lambda)\right) \frac{\beta(\lambda)-Y / \lambda}{|\beta(\lambda)-Y / \lambda|},
\end{aligned}
$$

and therefore

$$
\left|\frac{\partial h}{\partial \lambda}(\lambda, Y)\right| \leq\left|\beta(\lambda)+\lambda \beta^{\prime}(\lambda)\right|
$$

From this, one infers clearly that the mapping $(\lambda, Y) \mapsto H(\lambda, Y)$ is globally Lipschitz on $\mathbb{R} \times \mathbb{R}$. Writing

$$
Y\left(t, \lambda_{1}\right)-Y\left(t, \lambda_{2}\right)=\int_{0}^{t}\left(H\left(\lambda_{1}, Y_{1}\right)-H\left(\lambda_{2}, Y_{2}\right)\right)(t) d t+\left(\lambda_{1}-\lambda_{2}\right) X_{0} ;
$$

we conclude that

$$
\forall t \in(0, T), \quad\left|Y\left(t, \lambda_{1}\right)-Y\left(t, \lambda_{2}\right)\right| \leq K(T)\left|\lambda_{1}-\lambda_{2}\right|,
$$

thanks to an invocation of Gronwall lemma.

\subsection{Existence and uniqueness of the dynamical potential}

Since the non-linearity of problem $(23)$ appears in the transmission condition $(23 \mathrm{c})$, we are going to rewrite it on the surface $\Gamma$ using the Steklov-Poincare operators, in the same manner as we did in the previous section for the static model. We first prove the following property:

Lemma 3.3. The operator $\Lambda_{\mathrm{e}}+\Lambda_{\mathrm{c}}$ is positive, selfadjoint and invertible from $H^{1 / 2}(\Gamma)$ into $H^{-1 / 2}(\Gamma)$. The operator

$$
\mathcal{B}:=\operatorname{Id}+\Lambda_{\mathrm{e}}^{-1} \Lambda_{\mathrm{c}}
$$

is therefore invertible, from $H^{1 / 2}(\Gamma)$ into itself.

Moreover, define the domain $D\left(\Lambda_{\mathrm{c}} \mathcal{B}^{-1}\right)$ as

$$
D\left(\Lambda_{\mathrm{c}} \mathcal{B}^{-1}\right)=\left\{\varphi \in H^{1 / 2}(\Gamma): \Lambda_{\mathrm{c}} \mathcal{B}^{-1} \varphi \in L^{2}(\Gamma)\right\}
$$

The operator $\left(\Lambda_{\mathrm{c}} \mathcal{B}^{-1}, D\left(\Lambda_{\mathrm{c}} \mathcal{B}^{-1}\right)\right)$ is $m$-accretive (more precisely $D\left(\Lambda_{\mathrm{c}} \mathcal{B}^{-1}\right)=H^{1}(\Gamma)$ ). 
Proof. That $\Lambda_{\mathrm{e}}+\Lambda_{\mathrm{c}}$ is invertible is an easy consequence of the fact that the operator $\Lambda_{\mathrm{e}}$ is a positive, selfadjoint and invertible operator while $\Lambda_{\mathrm{c}}$ is non-negative and selfadjoint. Thus, $\Lambda_{\mathrm{e}}+\Lambda_{\mathrm{c}}$ is also a positive selfadjoint invertible operator.

Let $\varphi \in D\left(\Lambda_{\mathrm{c}} \mathcal{B}^{-1}\right)$. Then, by definition (and invertibility) of $\mathcal{B}\left(\right.$ i.e. $\left.\operatorname{Id}=\left(\operatorname{Id}+\Lambda_{\mathrm{e}}^{-1} \Lambda_{\mathrm{c}}\right) \mathcal{B}^{-1}\right)$ we have

$$
\begin{aligned}
\left\langle\Lambda_{\mathrm{c}} \mathcal{B}^{-1} \varphi, \varphi\right\rangle & =\left\langle\Lambda_{\mathrm{c}} \mathcal{B}^{-1} \varphi, \varphi\right\rangle \\
& =\left\langle\Lambda_{\mathrm{c}} \mathcal{B}^{-1} \varphi, \mathcal{B}^{-1} \varphi\right\rangle+\left\langle\Lambda_{\mathrm{c}} \mathcal{B}^{-1} \varphi, \Lambda_{\mathrm{e}}^{-1} \Lambda_{\mathrm{c}} \mathcal{B}^{-1} \varphi\right\rangle \\
& =\left\langle\Lambda_{\mathrm{c}} \mathcal{B}^{-1} \varphi, \mathcal{B}^{-1} \varphi\right\rangle+\left\langle\Lambda_{\mathrm{e}} \Lambda_{\mathrm{e}}^{-1} \Lambda_{\mathrm{c}} \mathcal{B}^{-1} \varphi, \Lambda_{\mathrm{e}}^{-1} \Lambda_{\mathrm{c}} \mathcal{B}^{-1} \varphi\right\rangle \\
& \geq 0 .
\end{aligned}
$$

$\Lambda_{\mathrm{c}} \mathcal{B}^{-1}$ is therefore accretive. Let $f \in L^{2}(\Gamma)$, let $\lambda>0$ and let $U$ be the unique solution in $P H_{0}^{1}(\Omega)$ to the following problem:

$$
\begin{aligned}
& -\Delta U=0, \text { in } \mathcal{O}_{\mathrm{e}} \cup \mathcal{O}_{\mathrm{c}}, \quad U_{\left.\right|_{\partial \Omega}}=0, \\
& \sigma_{\mathrm{e}} \partial_{\mathbf{n}} U_{\left.\right|_{\Gamma^{+}}}=\sigma_{\mathrm{c}} \partial_{\mathbf{n}} U_{\left.\right|_{\Gamma^{-}}}, \\
& \lambda \sigma_{\mathrm{c}} \partial_{\mathbf{n}} U_{\left.\right|_{\Gamma^{-}}}+U_{\left.\right|_{\Gamma^{-}}}-U_{\left.\right|_{\Gamma^{+}}}=f .
\end{aligned}
$$

Therefore, setting $v:=U_{\left.\right|_{\Gamma^{-}}}-U_{\left.\right|_{\Gamma^{+}}}, v$ satisfies

$$
v+\lambda \Lambda_{\mathrm{c}} \mathcal{B}^{-1} v=f
$$

In addition, since $\Lambda_{\mathrm{c}} \mathcal{B}^{-1}$ is nonnegative, we have $\|v\|_{L^{2}(\Gamma)} \leq\|f\|_{L^{2}(\Gamma)}$. Therefore, $\Lambda_{\mathrm{c}} \mathcal{B}^{-1}$ is $m$-accretive.

Lemma 3.4. Let $U_{0}$ and $X_{0}$ be two enough regular functions defined respectively in $\Omega$ and on $\Gamma$, and recall that $\widetilde{S}_{\mathrm{m}}(t, \lambda):=S_{\mathrm{L}}+\left(S_{\mathrm{ir}}-S_{\mathrm{L}}\right) X(t, \lambda)$ is defined in 22 . Finding the solution $(U, X)$ to problem (23)-24], if it exists, is equivalent to finding $\left(u_{\mathrm{e}}, u_{\mathrm{c}}, X\right)$, with $u_{\mathrm{e}}=U_{\mid \Gamma^{+}}$and $u_{\mathrm{c}}=U_{\mid \Gamma^{-}}$satisfying:

$$
\begin{aligned}
& u_{\mathrm{e}}=u_{\mathrm{c}}-v, \\
& u_{\mathrm{c}}=\mathcal{B}^{-1}\left(v-\Lambda_{\mathrm{e}}^{-1} \Lambda_{0} g\right),
\end{aligned}
$$

where $v$ is the solution to

$$
\begin{aligned}
& C_{\mathrm{m}} \partial_{t} v+\Lambda_{\mathrm{c}} \mathcal{B}^{-1} v+\widetilde{S}_{\mathrm{m}}(t, v) v=G, \\
& v(0, \cdot)=\varphi
\end{aligned}
$$

with $\varphi$ and $G$ being defined as

$$
\varphi=U_{\left.0\right|_{\Gamma^{+}}}-U_{\left.0\right|_{\Gamma^{-}}}, \quad G:=\Lambda_{\mathrm{c}} \mathcal{B}^{-1} \Lambda_{\mathrm{e}}^{-1} \Lambda_{0} g
$$

and where, writing $\lambda=v(t, s)$, a.e. $(t, s) \in(0, T) \times \Gamma, X$ satisfies

$$
\left\{\begin{array}{l}
\partial_{t} X(t, \lambda)=\max \left(\frac{\beta(\lambda)-X(t, \lambda)}{\tau_{\mathrm{ep}}} ; \frac{\beta(\lambda)-X(t, \lambda)}{\tau_{\mathrm{res}}}\right), \quad \forall t>0, \\
X(0, \lambda)=X_{0} .
\end{array}\right.
$$

$\mathrm{RR} \mathrm{n}^{\circ} 8005$ 
Proof. The lemma is a straightforward consequence of the definition of the Steklov-Poincare operators $\Lambda_{c}, \Lambda_{e}, \Lambda_{0}$, and of the invertibility of $\Lambda_{\mathrm{e}}$. Indeed, condition (23b), that is the continuity of the flux across $\Gamma$, boils down to

$$
\Lambda_{\mathrm{e}} u_{\mathrm{e}}+\Lambda_{0} g+\Lambda_{\mathrm{c}} u_{\mathrm{c}}=0,
$$

from which, thanks to the invertibility of $\Lambda_{e}$, we infer that

$$
u_{\mathrm{e}}-u_{\mathrm{c}}=-\left(\mathcal{B} u_{\mathrm{c}}+\Lambda_{\mathrm{e}}^{-1} \Lambda_{0} g\right)=-v .
$$

In addition, by definition of $v$, we have

$$
\Lambda_{\mathrm{c}} u_{\mathrm{c}}=\Lambda_{\mathrm{c}} \mathcal{B}^{-1} v-\Lambda_{\mathrm{c}} \mathcal{B}^{-1} \Lambda_{\mathrm{e}}^{-1} \Lambda_{0} g=\Lambda_{\mathrm{c}} \mathcal{B}^{-1} v-G .
$$

Then, the transmission condition 23c) (multiplied by $(-1)$ ) reads (31), provided we recall that $\beta$ is an even function and that $\widetilde{S}_{m}$ is defined by 22 .

We now show that the evolution equations appearing in lemma 3.4 have a unique solution.

Theorem 3.5. Assume that $\beta$ satisfies $(20), G \in L^{p}\left((0, T) ; L^{2}(\Gamma)\right)$ for some $p>1$, and that $\varphi \in L^{2}(\Gamma)$ is given. Let $X_{0} \in L^{\infty}(\Gamma)$ such that $0 \leq X_{0} \leq 1$ on $\Gamma$, and let $\widetilde{S}_{\mathrm{m}}$ be defined as in (22).

Then, there exists a unique function $v \in C\left([0, T] ; L^{2}(\Gamma)\right)$, mild solution to the system

$$
\left\{\begin{array}{l}
C_{\mathrm{m}} \partial_{t} v+\Lambda_{\mathrm{c}} \mathcal{B}^{-1} v+\widetilde{S}_{\mathrm{m}}(t, v) v=G, \quad \forall t \in(0, T) \\
v(0)=\varphi
\end{array}\right.
$$

where $\widetilde{S}_{\mathrm{m}}$ is given by 22 , and writing $\lambda=v(t, s)$, a.e $(t, s) \in(0, T) \times \Gamma$, one has

$$
\left\{\begin{array}{l}
\partial_{t} X(t, \lambda)=\max \left(\frac{\beta(\lambda)-X(t, \lambda)}{\tau_{\mathrm{ep}}} ; \frac{\beta(\lambda)-X(t, \lambda)}{\tau_{\mathrm{res}}}\right), \quad \forall t \in(0, T), \\
X(0, \lambda)=X_{0} .
\end{array}\right.
$$

Moreover, if $\varphi \in H^{1}(\Gamma)$ and $G \in W^{1,1}\left((0, T) ; L^{2}(\Gamma)\right)$, the above mild solution is a classical solution to 33 , in the sense that

$$
v \in C\left([0, T] ; H^{1}(\Gamma)\right) \cap C^{1}\left([0, T] ; L^{2}(\Gamma)\right) .
$$

Proof. To simplify the notations we denote by $\mathcal{A}$ the operator

$$
\mathcal{A}=\frac{1}{C_{\mathrm{m}}} \Lambda_{\mathrm{c}} \mathcal{B}^{-1} .
$$

In a first step, we are going to show existence and uniqueness of a mild solution to 33 in $C\left([0, T] ; L^{2}(\Gamma)\right)$. Thanks to lemma 3.2 , we know that for any $\lambda \in \mathbb{R}$, the solution to

$$
\left\{\begin{array}{l}
\partial_{t} X(t, \lambda)=\max \left(\frac{\beta(\lambda)-X(t, \lambda)}{\tau_{\mathrm{ep}}} ; \frac{\beta(\lambda)-X(t, \lambda)}{\tau_{\mathrm{res}}}\right), \forall t>0 \\
\left.X\right|_{t=0}=X_{0}
\end{array}\right.
$$


exists and belongs to $C^{1}([0, T])$. Moreover, $0 \leq X \leq 1$, and the mappings $\lambda \mapsto X$ and $\lambda \mapsto \lambda X$ are Lipschitz. Now, upon setting

$$
\mathcal{F}(t, v):=-\widetilde{S}_{\mathrm{m}}(t, v) v+G=-\left(S_{\mathrm{L}}+\left(S_{\mathrm{ir}}-S_{\mathrm{L}}\right) X(t, v)\right) v+G,
$$

solving equations (33) is equivalent to finding $v \in C\left([0, T] ; L^{2}(\Gamma)\right)$ solution to the following equation, which is the mild version of equation $(33)$ :

$$
v=\mathrm{e}^{-t \mathcal{A}} \varphi+\frac{1}{C_{\mathrm{m}}} \int_{0}^{t} \exp (-(t-\tau) \mathcal{A}) \mathcal{F}(\tau, v) d \tau .
$$

Thanks to inequality 26 of lemma 3.2 it is clear that the mapping

$$
v \mapsto \mathcal{F}(\cdot, v)
$$

is Lipschitz from the space

$$
\mathbb{E}:=C\left([0, T] ; L^{2}(\Gamma)\right)
$$

into itself. This means that there exists $K_{1}>0$ such that for any $v, w \in C\left([0, T] ; L^{2}(\Gamma)\right)$ we have

$$
\|\mathcal{F}(\cdot, v)-\mathcal{F}(\cdot, w)\|_{L^{\infty}\left((0, T) ; L^{2}(\Gamma)\right)} \leq K_{1}\|v-w\|_{L^{\infty}\left((0, T) ; L^{2}(\Gamma)\right)} .
$$

We shall endow the space $\mathbb{E}$ with the norm

$$
\|\psi\|_{\mathbb{E}}:=\sup _{t \in[0, T]} \mathrm{e}^{-\alpha t}\|\psi(t, \cdot)\|_{L^{2}(\Gamma)}
$$

for some $\alpha>0$, which will be chosen below. If we set

$$
\Phi\left(U_{\mathrm{c}}\right)(t):=\mathrm{e}^{-t \mathcal{A}} \varphi+\frac{1}{C_{\mathrm{m}}} \int_{0}^{t} \exp (-(t-\tau) \mathcal{A}) \mathcal{F}\left(\tau, U_{\mathrm{c}}(\tau)\right) d \tau,
$$

then $\Phi: \mathbb{E} \longrightarrow \mathbb{E}$ is a continuous mapping. We shall check that, upon choosing $\alpha$ appropriately, it is a strict contraction. Thus, it has a unique fixed point, providing the unique solution of (35). Indeed

$$
\Phi\left(U_{\mathrm{c}}\right)(t)-\Phi(V)(t)=\int_{0}^{t} \exp \left(-\frac{t-\tau}{C_{\mathrm{m}}} \Lambda_{\mathrm{c}} \mathcal{B}^{-1}\right)\left[\mathcal{F}\left(\tau, U_{\mathrm{c}}\right)-\mathcal{F}(\tau, V)\right] d \tau .
$$

Since the operator $\Lambda_{\mathrm{c}} \mathcal{B}^{-1}$ is $m$-accretive, the operator $\mathcal{A}$ generates a contraction semi-group and the following estimate holds

$$
\left\|\mathrm{e}^{-(t-\tau) \mathcal{A}}\left[\mathcal{F}\left(\tau, U_{\mathrm{c}}\right)-\mathcal{F}(\tau, V)\right]\right\|_{L^{2}(\Gamma)} \leq\left\|\left[\mathcal{F}\left(\tau, U_{\mathrm{c}}\right)-\mathcal{F}(\tau, V)\right]\right\|_{L^{2}(\Gamma)} .
$$

Therefore, we infer

$$
\begin{aligned}
\left\|\Phi\left(U_{\mathrm{c}}\right)(t)-\Phi(V)(t)\right\|_{L^{2}(\Gamma)} & \leq\left\|\int_{0}^{t}\right\| \mathcal{F}\left(\tau, U_{\mathrm{c}}\right)-\mathcal{F}(\tau, V) \|_{L^{2}(\Gamma)} d \tau \\
& \leq K_{1}\left\|U_{\mathrm{c}}-V\right\|_{\mathbb{E}} \int_{0}^{t} \mathrm{e}^{\alpha \tau} d \tau \\
& \leq C(p) K_{1} \alpha^{-1} \mathrm{e}^{\alpha t}\left\|U_{\mathrm{c}}-V\right\|_{\mathbb{E}}
\end{aligned}
$$

from which we conclude that

$$
\left\|\Phi\left(U_{\mathrm{c}}\right)-\Phi(V)\right\|_{\mathbb{E}} \leq \alpha^{-1} C(p) K_{1}\left\|U_{\mathrm{c}}-V\right\|_{\mathbb{E}} .
$$

RR n 8005 
This implies that for $\alpha$ large enough, the mapping $\Phi$ is a strict contraction on $\mathbb{E}$. Equation 33 has thus a unique mild solution in $C\left([0, T] ; L^{2}(\Gamma)\right)$.

Suppose now that $G$ belongs to $W^{1,1}\left((0, T) ; L^{2}(\Gamma)\right)$ and that $\varphi \in H^{1}(\Gamma)$. The mild solution given by formula 35 belongs to $C^{1}\left([0, T] ; L^{2}(\Gamma)\right)$ hence we infer

$$
\partial_{t} v+\widetilde{S}_{\mathrm{m}}(\cdot, v) v \in C\left([0, T] ; L^{2}(\Gamma)\right) .
$$

Therefore, setting $U_{\mathrm{c}}=U_{\mid \mathcal{O}_{\mathrm{c}}}$, we have

$$
\Delta U_{\mathrm{c}}(t, \cdot)=0, \operatorname{in} \mathcal{O}_{\mathrm{c}}, \quad \sigma_{\mathrm{c}} \partial_{n} U_{\mathrm{c}}(t, \cdot) \in L^{2}(\Gamma),
$$

from which we infer that $U_{\mathrm{c}}(t, \cdot) \in H^{3 / 2}\left(\mathcal{O}_{\mathrm{c}}\right)$. Hence we deduce that since the domains are smooth $U_{\mathrm{c}}(t, \cdot)_{\mid \Gamma^{-}}$belongs to $H^{1}(\Gamma)$. Similar reasoning for $U_{\mathrm{e}}=U_{\mid \mathcal{O}_{\mathrm{e}}}$ implies that $U_{\mathrm{e}}(t, \cdot)_{\mid \Gamma^{+}}$belongs to $H^{1}(\Gamma)$, and therefore the jump $v$ belongs to $C\left([0, T] ; H^{1}(\Gamma)\right)$, which ends the proof.

\section{Numerical simulations}

In this section, we provide some numerical results that show the consistency of our models. In order to solve both static and dynamical problems, we first present the finite-difference method on a cartesian grid adapted from the second-order scheme of Cisternino and Weynans [2]. Note that it is not the scope of this paper to prove rigorously that our scheme is of order two, however numerical simulations of Fig 3(b) seems to confirm this order. The rigorous numerical analysis of the method will be performed in a forthcoming work. Since the long-term goal of this work is to fit the models with the experimental data, and since experiments are performed with several thousands of cells per $\mathrm{cm}^{2}$, parallel computing will be necessary to solve the inverse problem. Therefore, we choose to use accurate schemes on cartesian grids, such as the scheme of 2], for which the accuracy has been shown and the parallelization has be already performed, despite Galerkin formulations might be more adapted to the single cell problem, since variational formulation holds.

\subsection{Spatial discretization}

We perform the discretization on a cartesian grid covering the domain $\Omega=\mathcal{O}_{\mathrm{e}} \cup \overline{\mathcal{O}_{\mathrm{c}}}$, which is a square domain of length $L$ ( $L$ equals $200 \mu \mathrm{m}$ for the computations, see Table11). The interface is described by a level-set function [16], which separates the extra- and intra-cellular domains by the use of a signed distance function $\varphi$. The normal to the interface $\mathbf{n}(x)$ outwardly directed from the inner to the outer of the cell is directly obtained by computing numerically $\nabla \varphi(x)$.

The grid spacing is denoted by $h$, and $N$ is the number of points such as

$$
N=L / h .
$$

For any $(i, j) \in N^{2}$ we denote by $M_{i j}$ the grid points defined by

$$
M_{i, j}=\left(x_{i}, y_{j}\right), \quad \text { where } \quad x_{i}=i h_{x}, y_{j}=j h_{y}, \quad \forall(i, j) \in N^{2} .
$$

The numerical approximation of the solution to the static or to the dynamical model at the point $\left(x_{i}, y_{j}\right)$ is generically denoted by $u_{i j}$.

Standard approximation of the Laplacian is used in the discretized domains $\mathcal{O}_{\mathrm{e}}$ and $\mathcal{O}_{\mathrm{c}}$ far from the interface, which is the cell membrane $\Gamma$. However, due to the jump conditions, a special treatment of the approximation of the Laplacian and of the computation of the fluxes is needed at the points nearing the cell membrane. 
If the intersection of the interface and $\left[M_{i j} M_{i+1 j}\right]$ exists, then we define the interface point $I_{i+1 / 2, j}=\left(\tilde{x}_{i+1 / 2, j}, y_{j}\right)$ as this intersection. We create two additional unknowns at this interface point, called interface unknowns, and denoted by $\tilde{u}_{i+1 / 2, j}^{\mathrm{e}}$ and $\tilde{u}_{i+1 / 2, j}^{\mathrm{c}}$. The interface point $I_{i, j+1 / 2}=\left(x_{i}, \tilde{y}_{i, j+1 / 2}\right)$ is similarly defined as the intersection of $\Gamma$ and the segment $\left[M_{i j} M_{i j+1}\right]$.

An example of the discretization method is given by Figure 2. On regular grid points, that are not neighboring the interface, the Laplacian is discretized with a standard centered second-order finite-difference scheme. A specific five points stencil including the interface points is used for neighboring points, as shown in Figure 2(a).

Figure 2(b) provides an example of the discretization of $\nabla U$ on both sides of the interface. The $x$-derivative of $U$ can be computed with second-order accuracy using a one-sided formula involving three grid points. For example we approximate the flux on the left (for instance exterior) side of the interface with the points $M_{i-1 j}, M_{i j}$ and $I_{i+1 / 2, j}$ by:

$$
\frac{\partial U}{\partial x}\left(\tilde{x}, y_{j}\right) \approx \frac{\left(u_{i-1 j}-\tilde{u}_{i+1 / 2, j}^{\mathrm{e}}\right)\left(x_{i}-\tilde{x}\right)}{h_{x}\left(x_{i-1}-\tilde{x}\right)}-\frac{\left(u_{i j}-\tilde{u}_{i+1 / 2, j}^{\mathrm{e}}\right)\left(x_{i-1}-\tilde{x}\right)}{h_{x}\left(x_{i}-\tilde{x}\right)},
$$

where for the sake of brevity, we have replaced $\tilde{x}_{i+1 / 2, j}$ by $\tilde{x}$. The $y$-derivative cannot be obtained in the same way, since there are no grid points aligned with the interface point in the $y$-direction. We therefore use a linear combination of $\left(\partial_{y} u\right)_{i j}$ and $\left(\partial_{y} u\right)_{i-1 j}$, defined respectively as second order approximations of the $y$-derivative on $M_{i j}$ and $M_{i-1 j}$. We obtain

$$
\frac{\partial U^{\mathrm{e}}}{\partial y}\left(\tilde{x}, y_{j}\right) \approx \frac{\tilde{x}-x_{i-1}}{h_{x}}\left(\partial_{y} u\right)_{i j}-\frac{\tilde{x}-x_{i}}{h_{x}}\left(\partial_{y} u\right)_{i-1 j}
$$

The formulas for $\left(\partial_{y} u\right)_{i j}$ and $\left(\partial_{y} u\right)_{i-1 j}$ depend on the local configuration on the interface, but they are based on the same principle as for $(36)$. The scheme is stabilized by using a shifted $y$-stencil if two interface points are involved in the same flux discretization, as illustrated by Figure 2(c)

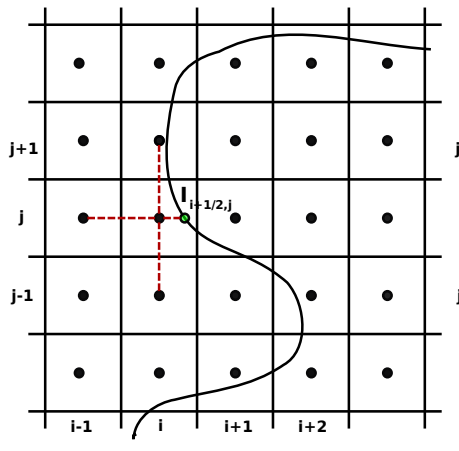

(a) Discretization of the Laplacian on the points at the interface.

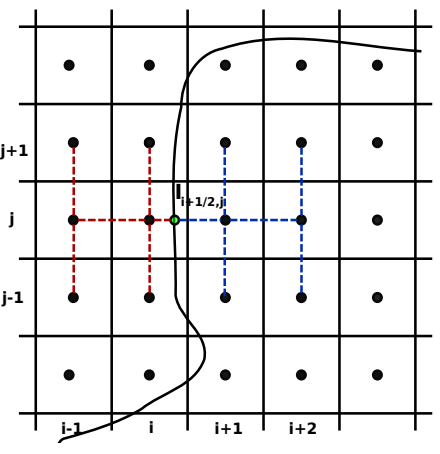

b) Discretization of $\nabla U$ at the interface: non-stabilized stencil.

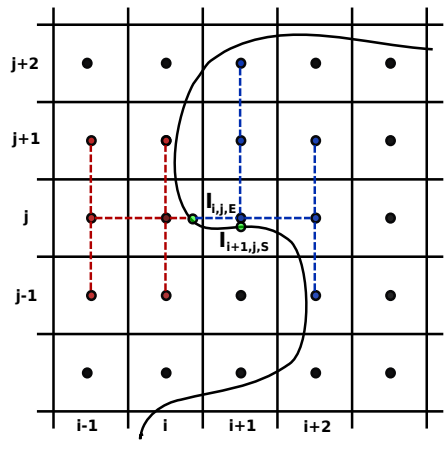

(c) Discretization of $\nabla U$ at the interface: stabilized stencil.

Figure 2: Discretizations of the Laplacian and of the gradient of $U$ at the interface. The first $y$-derivative stencil on the right side is shifted to avoid an ill-conditioned discretization.

\subsection{Accuracy of the finite difference method}

In order to show the accuracy of the numerical method, we compare both explicit and numerical solutions to the linear static problem (4), i.e without electroporation. This means that in (4c), $S_{\mathrm{m}}$ is constant equal to $S_{\mathrm{L}}$. 
Consider a domain composed of two concentric disks. The cell is the disk of radius $R_{1}$, centered at 0 . The domain $\Omega$ is the disk with the same center as the cell, and whose radius $R_{2}$ is stricly greater than $R_{1}$. The boundary data $g$ equals $E R_{2} \cos \theta$, such that the cell is embbeded in a uniform electric field of magnitude $E$ in the $x$-direction. The exact solution $\widetilde{U}$ to $(4)$ is then explicitely given by

$$
\begin{aligned}
\forall(r, \theta) \in\left(R_{1}, R_{2}\right) \times[0,2 \pi], & \widetilde{U}_{\mathrm{e}}(r, \theta)=\left(\alpha_{\mathrm{e}} r+\beta_{\mathrm{e}} r^{-1}\right) \cos \theta, \\
\forall(r, \theta) \in\left(0, R_{1}\right) \times[0,2 \pi], & \widetilde{U}_{\mathrm{c}}(r, \theta)=\alpha_{\mathrm{c}} r \cos \theta,
\end{aligned}
$$

where $\alpha_{\mathrm{e}}, \beta_{\mathrm{e}}$, and $\alpha_{\mathrm{c}}$ are given by

$$
\begin{aligned}
& \alpha_{\mathrm{c}}=\left(\left(\frac{\sigma_{\mathrm{c}}}{S_{\mathrm{L}} R_{1}}+1+\frac{\sigma_{\mathrm{c}}}{\sigma_{\mathrm{e}}}\right) R_{2}+\left(\frac{\sigma_{\mathrm{c}}}{S_{\mathrm{L}} R_{1}}+1-\frac{\sigma_{\mathrm{c}}}{\sigma_{\mathrm{e}}}\right) \frac{R_{1}^{2}}{R_{2}}\right)^{-1} g, \\
& \alpha_{\mathrm{e}}=\frac{1}{2}\left(\frac{\sigma_{\mathrm{c}}}{S_{\mathrm{L}} R_{1}}+1+\frac{\sigma_{\mathrm{c}}}{\sigma_{\mathrm{e}}}\right) \alpha_{\mathrm{c}}, \\
& \beta_{\mathrm{e}}=\frac{1}{2}\left(\frac{\sigma_{\mathrm{c}}}{S_{\mathrm{L}} R_{1}}+1-\frac{\sigma_{\mathrm{c}}}{\sigma_{\mathrm{e}}}\right) \alpha_{\mathrm{c}} R_{1}^{2} .
\end{aligned}
$$

This analytic solution is projected on the edges of the square $Q_{L}$ centered at 0 , and whose characteristic length $L$ satisfies $R_{1}<L / 2<L \sqrt{2} / 2<R_{2}$ (see Fig 3(a)).

In order to verify the accuracy of the space discretization, at least in this configuration, we solve numerically problem (4) in the square $Q_{L}$, with the trace $\left.\widetilde{U}\right|_{\partial Q_{L}}$ of the analytic solution $\widetilde{U}$ on the edges of $Q_{L}$ as Dirichlet boundary condition for different grid spacings $h$. We set the parameters equal to

$$
R_{2}=150 \mu \mathrm{m}, \quad R_{1}=50 \mu \mathrm{m}, \quad L=200 \mu \mathrm{m}, \quad E=400 \mathrm{~V} / \mathrm{cm},
$$

the electric parameters being given by Table 1. These parameters come from the values given by DeBruin and Krassowska, see Table 1 of [3].

We then compare the numerical solution $U_{h}$ to $\widetilde{U}$. The relative error is computed using both grid and interface points:

$$
E\left(\widetilde{U}, U_{h}\right):=\frac{\| U_{h}-\left.\widetilde{U}\right|_{L^{2}(\Omega)}+\left|U_{h}-\widetilde{U}\right|_{L^{2}(\Gamma)}}{\|\left.\widetilde{U}\right|_{L^{2}(\Omega)}+|\widetilde{U}|_{L^{2}(\Gamma)}} .
$$

We denote by $h_{r}$ the relative grid spacing defined by

$$
h_{r}=h / L
$$

Figure 3(b) shows the behavior of $E\left(\widetilde{U}, U_{h}\right)$ with respect to $h_{r}$ : the space discretization is of order two for this specific case. Note Cisternino and Weynans [2 have shown the second order accuracy of their method, therefore despite we have adapted the scheme to our problem, we are confident in the good accuracy of the numerical method.

\subsection{Computation of the non-linear static model}

When solving the static equation (4), if one uses the following naive iterative method:

$$
S_{\mathrm{m}}\left(\left[U^{n}\right]\right)\left[U^{n+1}\right]=\sigma_{\mathrm{c}} \partial_{\mathbf{n}} U_{\mathrm{c}}^{n+1},
$$


Domain of calculation of the analytic solution

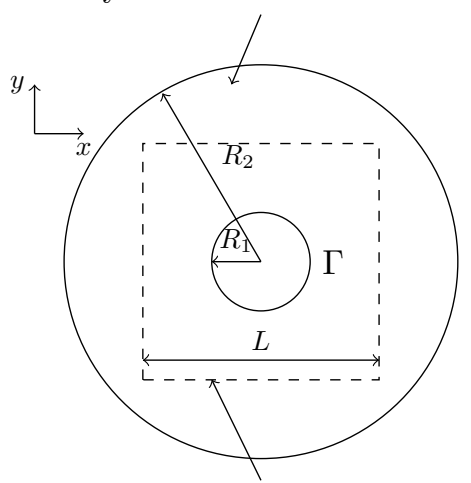

Computational domain

(a) Domains of calculation of the analytic and of the numerical solutions.

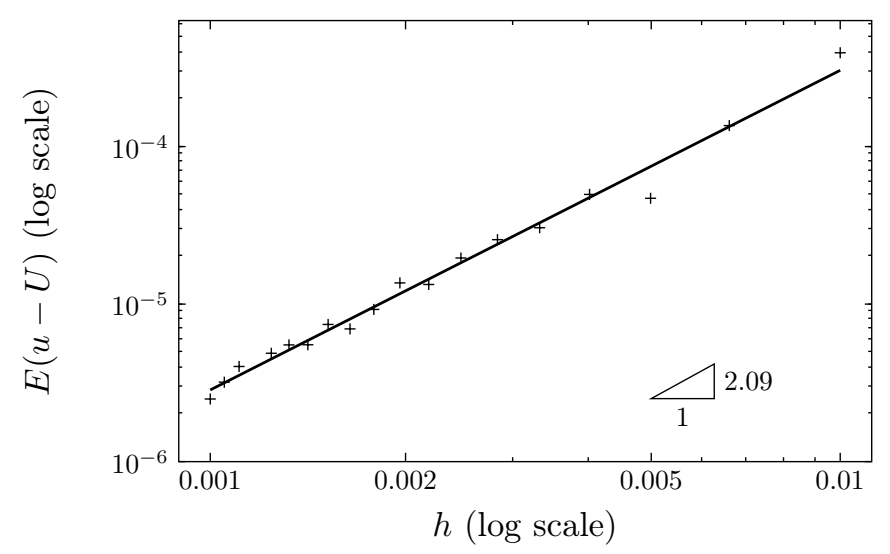

(b) Log-log diagram of the error $E\left(\widetilde{U}, U_{h}\right)$ given by 39 with respect to $h_{r}$.

Figure 3: Numerical estimation of the order of accuracy of the method. The analytic solution to the linear problem is calculated in concentric circular domains. The restriction of this solution to the boundary of the computational domain (dashed line) provides the Dirichlet data for the numerical solution. Relative error between the two solutions with respect to the grid spacing is plotted in Figure $3(\mathrm{~b})$.

one finds that the iterative scheme oscillates between two values. This might be a consequence of the fact that the membrane conductivity takes its extreme values $S_{\mathrm{L}}$ and $S_{\text {ir }}$ instead of reaching an intermediate state. Another issue might be that the mapping $\mathcal{L}_{g}$ defined by

$$
\mathcal{L}_{g}: v \mapsto u,
$$


where $u \in P H^{1}(\Omega)$ is solution to

$$
\left\{\begin{array}{l}
\Delta u=0, \quad \text { in } \mathcal{O}_{\mathrm{e}} \cup \mathcal{O}_{\mathrm{c}}, \quad u_{\left.\right|_{\partial \Omega}}=g, \\
{\left[\sigma \partial_{\mathbf{n}} u\right]_{\Gamma}=0, \quad S_{\mathrm{m}}\left([v]_{\Gamma}\right)[u]_{\Gamma}=\left.\sigma_{\mathrm{c}} \partial_{\mathbf{n}} u\right|_{\Gamma},}
\end{array}\right.
$$

is not a contraction, since the Lipschitz constant of $S_{\mathrm{m}}$ is of order $k_{\mathrm{ep}} S_{\mathrm{m}} \gg 1$.

We use a modified mapping $\mathcal{L}_{\rho, g}$ :

$$
\mathcal{L}_{\rho, g}: v \mapsto u,
$$

where $u \in P H^{1}(\Omega)$ is solution to

$$
\left\{\begin{array}{l}
\Delta u=0, \quad \text { in } \mathcal{O}_{\mathrm{e}} \cup \mathcal{O}_{\mathrm{c}}, \quad u_{\mid \partial \Omega}=g, \\
{\left[\sigma \partial_{\mathbf{n}} u\right]_{\Gamma}=0, \quad[u]_{\Gamma}+\rho S_{\mathrm{m}}\left([v]_{\Gamma}\right)[u]_{\Gamma}-\left.\rho \sigma_{\mathrm{c}} \partial_{\mathbf{n}} u\right|_{\Gamma}=[v]_{\Gamma},}
\end{array}\right.
$$

where $\rho$ is a small positive parameter chosen so as $\mathcal{L}_{\rho, g}$ be a contractive operator. The following iteration process is used:

$$
U^{0} \text { given, } \quad \text { and for any } n \geq 0 \quad U^{n+1}=\mathcal{L}_{\rho, g}\left(U^{n}\right) .
$$

For the simulations we set the stopping criterion of the scheme at $\gamma=10^{-12}$, meaning that the numerical solution is obtained when the relative error $E\left(U^{n+1}, U^{n}\right)$ is smaller that $\gamma$.

\subsubsection{Influence of the parameter $S_{\mathrm{ir}}$}

The parameter $S_{\mathrm{ir}}$, which is the conductivity of the fully "electroporated" membrane is hardly measurable by the experiments. It is therefore important to investigate its influence on the model. Figure 4 shows that $S_{\text {ir }}$ has a little influence on the membrane conductivity $S_{\mathrm{m}}$, as the value of $X$ counter-balance the variation of $S_{\mathrm{ir}}$. Therefore, the numerical criterion to define the electroporation should involve the parameter $S_{\mathrm{m}}$.

\subsubsection{Comparison with the model of Ivorra, Mir and Villemejeane}

We compare our results with the simulations of Ivorra et al. by studying the influence of the extracellular medium conductivity on the membrane conductivity. In Figure 5 , we show results similar to those presented in Figure 7 of [10. Note that to perform their simulations, Ivorra et al. have multiplied by ten the membrane thickness. This is the reason why their permeabilizing field is of order of magnitude of $2 \mathrm{MV} / \mathrm{m}$, which is much higher than the magnitude used in the experiments, typically [12, 13, 6] to the range 20 to $30 \mathrm{kV} / \mathrm{m}$. In contrast, our model provides more realistic conditions of electropermeabilization.

\subsection{Computation of the dynamical problem}

\subsubsection{Time-discretization of the model}

The time-derivative $\partial_{t}[U]$ of 23 is discretized using the following scheme:

$$
C_{\mathrm{m}} \frac{[U]^{n+1}-[U]^{n}}{d t}-\sigma_{\mathrm{c}} \partial_{\mathbf{n}} U_{\mathrm{c}}^{n+1}+\widetilde{S}_{\mathrm{m}}\left(t^{n},[U]^{n}\right)[U]^{n}=0 .
$$

The Runge-Kutta method of order 4 is used to compute the variable $X$, with time steps $d t$. 


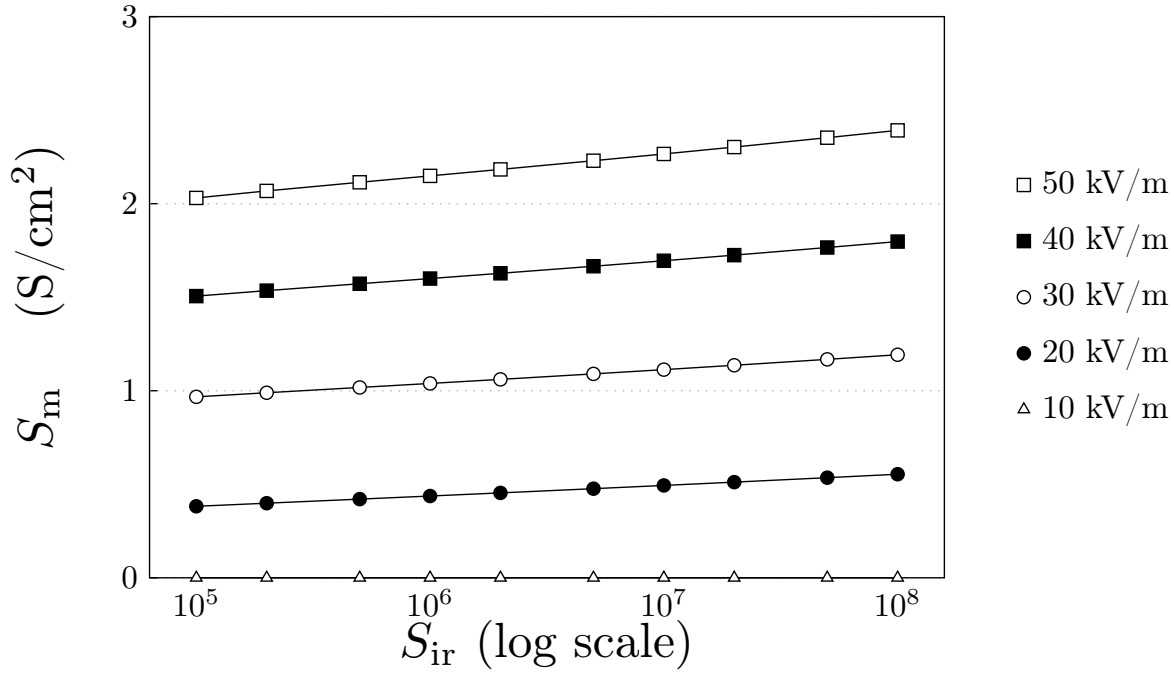

Figure 4: Membrane surface conductivity $S_{\mathrm{m}}$ at the pole of the cell $(\theta=0)$ obtained by solving the static equation with different values of $S_{\text {ir }}$ for pulse magnitudes from 10 to $50 \mathrm{kV} / \mathrm{m}$ (the numerical values of the other parameters are given in Table 1).

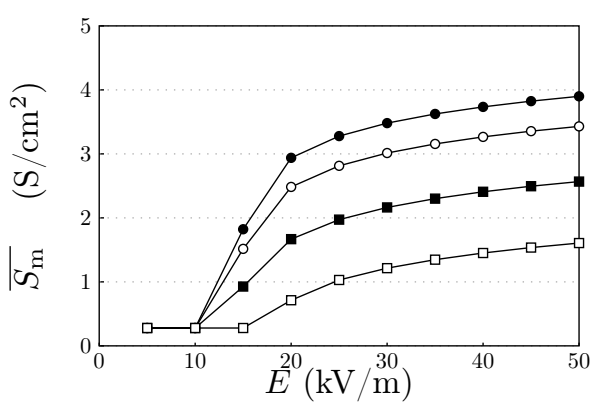

(a) Average value $\overline{S_{\mathrm{m}}}$ of $S_{\mathrm{m}}$ over $\Gamma$.

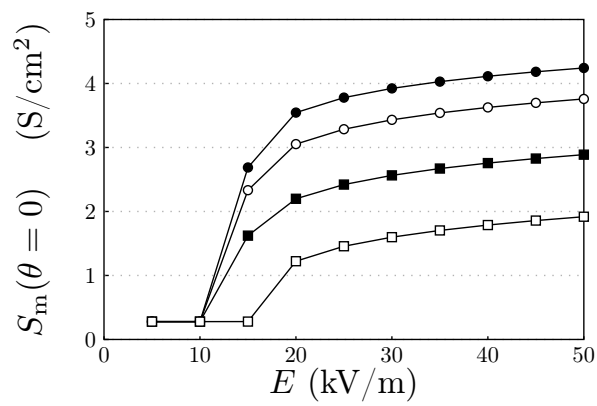

(b) Pointwise value of $S_{\mathrm{m}}$ at the cell pole.

Figure 5: Non-linear membrane conductivity of the static model for 4 different extracellular conductivity: $\sigma_{\mathrm{e}}=1 \mathrm{~S} / \mathrm{m}(\bullet), \sigma_{\mathrm{e}}=0.1 \mathrm{~S} / \mathrm{m}(\circ), \sigma_{\mathrm{e}}=0.01 \mathrm{~S} / \mathrm{m}(\boldsymbol{\square}), \sigma_{\mathrm{e}}=0.001 \mathrm{~S} / \mathrm{m}(\square)$. 
Table 1: Parameters set to fit to the results given by [14, 3]. (EP stands for electropermeabilization, and EPd stands for electropermeabilized).

\begin{tabular}{llll}
\hline Variable & Symbol & Value & Unit \\
\hline Biological parameters: & & & \\
Extracellular conductivity & & & \\
Intracellular conductivity & $\sigma_{\mathrm{e}}$ & 5 & $\mathrm{~S} / \mathrm{m}$ \\
Capacitance & $\sigma_{\mathrm{c}}$ & 0.455 & $\mathrm{~S} / \mathrm{m}$ \\
Membrane surface conductivity & $C_{\mathrm{m}}$ & $9.5 \times 10^{-3}$ & $\mathrm{~F} / \mathrm{m}^{2}$ \\
Cell radius & $S_{\mathrm{L}}$ & 1.9 & $\mathrm{~S} / \mathrm{m}^{2}$ \\
Membrane thickness & $r$ & 50 & $\mu \mathrm{m}$ \\
& $\delta$ & 5 & $\mathrm{~nm}$ \\
\hline Specific parameters of the model: & & & \\
EP threshold & $V_{\mathrm{rev}}$ & 1,5 & $\mathrm{~V}$ \\
EP switch speed & $k_{\mathrm{ep}}$ & 40 & $\mathrm{~V}-1$ \\
EP characteristic time & $\tau_{\mathrm{ep}}$ & $1 \times 10^{-6}$ & $\mathrm{~s}$ \\
Resealing characteristic time & $\tau_{\mathrm{res}}$ & $1 \times 10^{-3}$ & $\mathrm{~S}$ \\
EPd membrane surface conductivity & $S_{\mathrm{ir}}$ & $2.5 \times 10^{8}$ & $\mathrm{~S} / \mathrm{m}^{2}$ \\
& & & \\
\hline Numerical parameters: & & & \\
Simulation box size & & & \\
Grid points (each side) & $L$ & 200 & $\mu \mathrm{m}$ \\
Time step & $N$ & 50 & \\
Pulse duration & $N_{T}$ & 7500 & $\mathrm{~ns}$ \\
$\begin{array}{l}\text { Duration of simulation } \\
\text { Number of time steps }\end{array}$ & $T_{\mathrm{p}}$ & 100 & \\
& & & \\
\hline
\end{tabular}


Figure 6(a) and 6(b) show the numerical results at $t=100 \mu \mathrm{s}$ using the parameters of Table 1 . In order to visualize the membrane electropermeabilization, we depict it with boxes, which are colored and sized according to the values of $\widetilde{S}_{\mathrm{m}}$ at each point of $\Gamma$ and at $t=100 \mu \mathrm{s}$. We emphasize this is a visualization artefact: in our model, the cell membrane is a surface without any thickness.
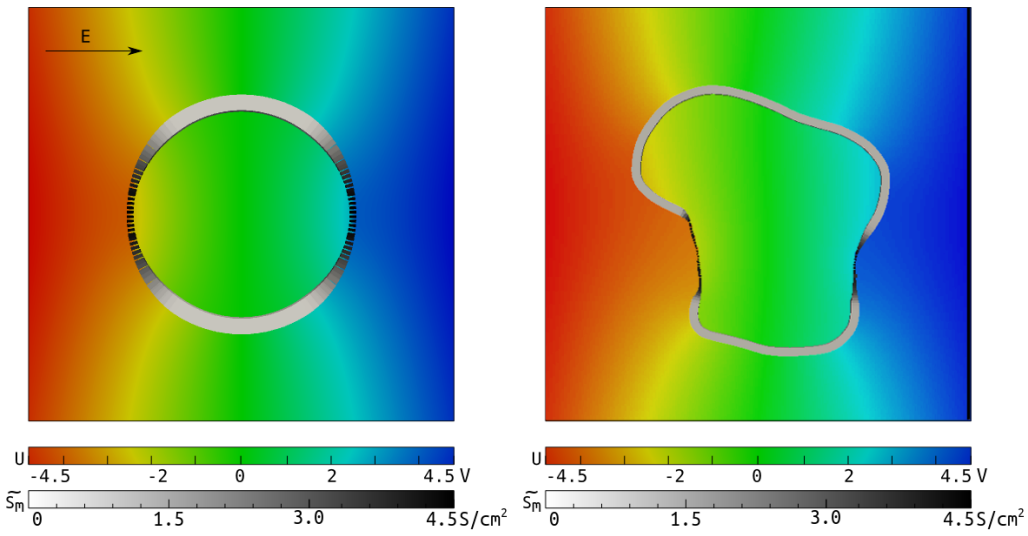

(a) Solution to the dynamical model for (b) Solution to the dynamical model for a circular cell. a smooth non-convex cell shape.

Figure 6: Solution to the dynamical problem with two different cell shapes at $t=100 \mu \mathrm{s}$. The numerical parameters are given by Table 1. Fig. 6(b) shows that the electropermeabilized regions depend on the shape and orientation of $\Gamma$.

\subsubsection{Main parameters influence}

The key parameters of the model define the electropermeabilization coefficient $\widetilde{S}_{\mathrm{m}}$, that is $k_{\mathrm{ep}}$, $V_{\text {rev }}, S_{\text {ir }}$ and the characteristic times $\tau_{\text {ep }}$ and $\tau_{\text {res. }}$. A numerical sensitivity analysis was led to determine how the behavior of the solution with respect to a variation of each specific parameter, as shown on Figure 7. All the parameters defining the function $\beta$ have a very small influence on the average $\bar{X}$ of $X$ over the cell membrane. Even for small values of $k_{\mathrm{ep}}$, the values of $\bar{X}$ are only modified by a factor 2 (Figure 7(a). On the other hand, the "fully electroporated" membrane conductivity $S_{\mathrm{ir}}$, which was first taken as $\left(\sigma_{\mathrm{c}}+\sigma_{\mathrm{e}}\right) /(2 \delta)$, affects greatly the order of magnitude of $\bar{X}$, changing from $10^{-6}$ to $10^{-2}$ (Figure $7(\mathrm{~d})$. Therefore, as for the static case the relevant quantity to observe the phenomenon is $\widetilde{S}_{\mathrm{m}}$.

\subsubsection{Comparison with the model of Neu, Krassowska, and Debruin}

The main difference between the model of Neu, Krassowska, et al. and ours resides in the addition of an electroporation current $I_{\mathrm{ep}}=N_{\mathrm{ep}} i_{\mathrm{ep}}$, instead of a direct description of the variations of the surface membrane conductivity $\widetilde{S}_{\mathrm{m}}$.

The equation satisfied by the transmembrane voltage in the model of Neu, Krassowska, et al. 3] reads

$$
-\sigma_{\mathrm{c}} \partial_{n} U_{\mathrm{c}}=C_{\mathrm{m}} \partial_{t}[U]+S_{\mathrm{L}}[U]+N_{\mathrm{ep}} i_{\mathrm{ep}}
$$

where the ionic reversal currents have been neglected. $N_{\text {ep }}$ is the pore density, obeying the 


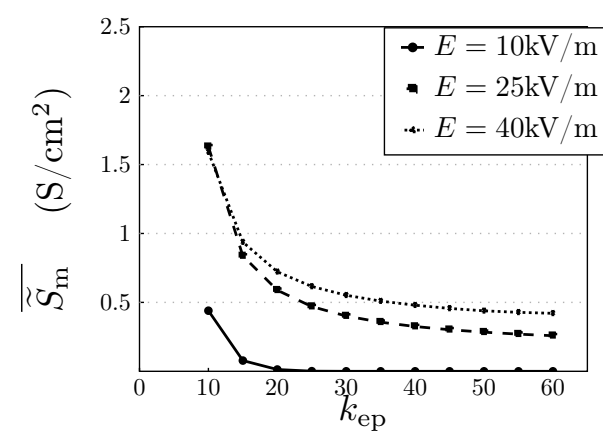

(a) Influence of EP switch coefficient $k_{\mathrm{ep}}$.

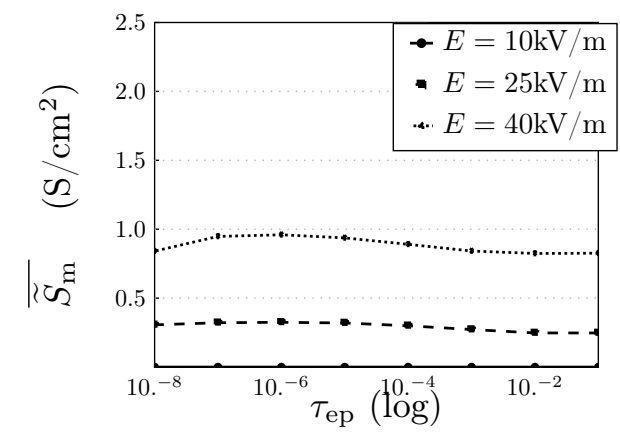

(c) Influence of the characteristic time $\tau_{\mathrm{ep}}$.

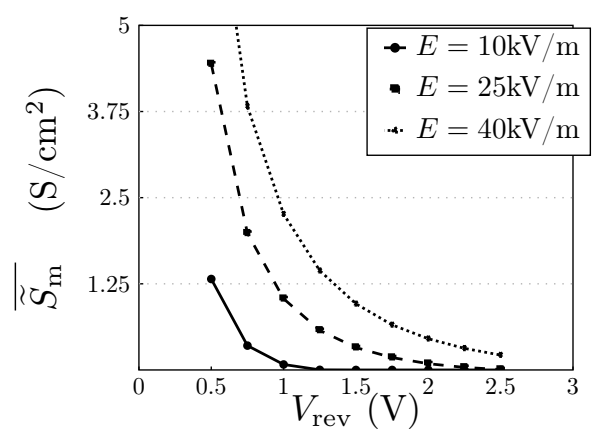

(b) Influence of threshold voltage $V_{\text {rev }}$.

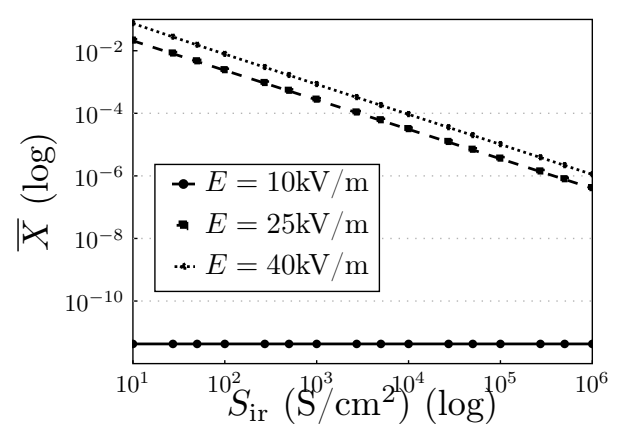

(d) Influence of the EPd surface conductivity $S_{\mathrm{ir}}$.

Figure 7: Influence of each parameter on the mean value $\widetilde{\widetilde{S}_{\mathrm{m}}}$ of $\widetilde{S}_{\mathrm{m}}$ (Fig. $7(\mathrm{a}) \mathrm{7( \textrm {b } )}$ 7(c) 7 , and on the mean value $\bar{X}$ of $X$ (Fig. $7(\mathrm{~d}))$ at $t=100 \mu \mathrm{s}$. Three magnitudes of electric pulses are considered: 10,25 and $40 \mathrm{kV} / \mathrm{m}$. 
ordinary differential equation (parameters are emphasized in bold):

$$
\frac{\mathrm{d} N_{\mathrm{ep}}}{\mathrm{d} t}=\boldsymbol{\alpha} \mathrm{e}^{\left([U] / \boldsymbol{V}_{\text {rev }}\right)}\left(1-\frac{N_{\mathrm{ep}}}{\boldsymbol{N}_{\mathbf{0}}} \mathrm{e}^{-\boldsymbol{q}\left([U] / \boldsymbol{V}_{\text {rev }}\right)^{2}}\right),
$$

and $i_{\mathrm{ep}}$ is the current flowing through a single pore:

$$
i_{\mathrm{ep}}\left(v_{\mathrm{m}}\right)=\frac{\pi \boldsymbol{r}_{\mathbf{m}}^{\mathbf{2}} \boldsymbol{\sigma} R T}{F \delta} \frac{v_{\mathrm{m}}\left(\mathrm{e}^{v_{\mathrm{m}}}-1\right)}{\frac{\boldsymbol{w}_{\mathbf{0}} \mathrm{e}^{\boldsymbol{w}_{\mathbf{0}}-\boldsymbol{n} v_{\mathrm{m}}}-\boldsymbol{n} v_{\mathrm{m}}}{\boldsymbol{w}_{\mathbf{0}}-\boldsymbol{n} v_{\mathrm{m}}} \mathrm{e}^{v_{\mathrm{m}}}-\frac{\boldsymbol{w}_{\mathbf{0}} \mathrm{e}^{\boldsymbol{w}_{\mathbf{0}}+\boldsymbol{n} v_{\mathrm{m}}}+\boldsymbol{n} v_{\mathrm{m}}}{\boldsymbol{w}_{\mathbf{0}}+\boldsymbol{n} v_{\mathrm{m}}}},
$$

with $v_{\mathrm{m}}=[U] \times F / R T$ the adimensionalized transmembrane voltage.

The numerical parameters for the model of Neu, Krassowska, et al. are those of Table 1 page 1215 of [3]. Our model reproduces qualitatively the behavior of $\triangle T M P$ as shown in Figure 8 . In Figure 9 , we show that the variation of the membrane current density $\widetilde{S}_{\mathrm{m}}[U]$ of our modeling is similar to the electroporation current density $N_{\mathrm{ep}} i_{\mathrm{ep}}$ of $[3$.

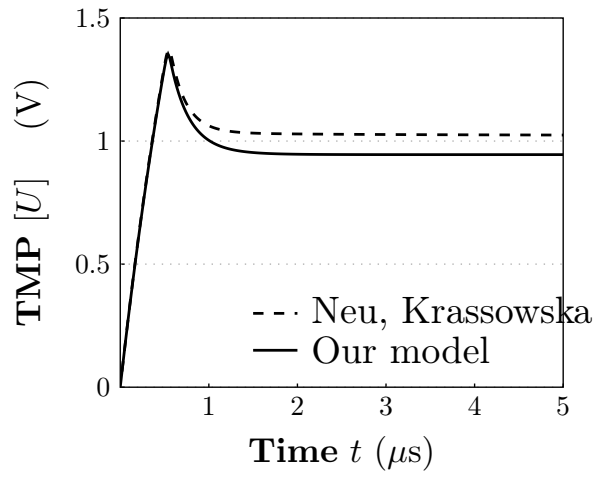

(a) Evolution of the $\triangle \mathrm{TMP}$ at the cell's pole.

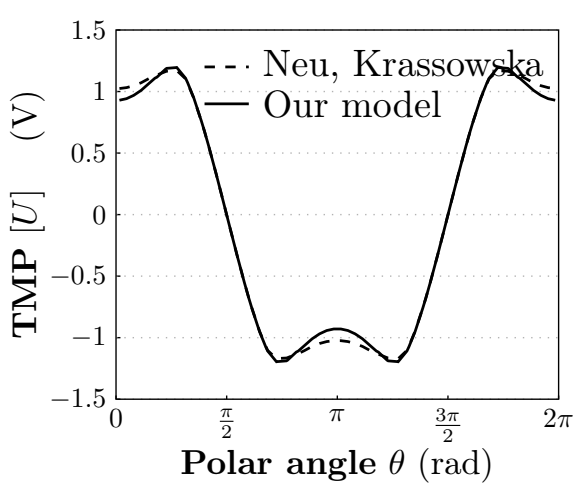

(b) Values of the $\triangle \mathrm{TMP}$ after $100 \mu$ s along the perimeter of the circular cell.

Figure 8: Comparison of the $\triangle \mathrm{TMP}$ obtained respectively with our model (solid lines) and with the model of Neu, Krassowska, et al. (dashed), with parameters from table 1 .

\subsubsection{Long-time behavior of the numerical solution to the dynamical model}

In this paragraph, we compare the long-time behavior of the solution $U_{\mathrm{dyn}}$ to the dynamic model for a constant pulse with the solution $U_{\text {stat }}$ to the static model.

Simulations are done in order to reach the time scale of the resealing characteristic time $\tau_{\text {res }}$ (Figure 10. A constant pulse $g$ is applied until the steady state of the dynamical system is reached. Observe that the stationary value of $\widetilde{S}_{\mathrm{m}}$, which is reached after about $500 \mu \mathrm{s}$ is lower than the value of $\widetilde{S}_{\mathrm{m}}$ a few tens of microseconds after the begining of the pulse delivery. For pulses of short duration, it is therefore important to simulate our new dynamical model instead of the steady model of Ivorra et al. [10.

\section{Conclusion}

In this paper, we introduce two models describing the electropermeabilization of a single cell. We first study the static model, which is inspired by Ivorra et al., and we show existence and 


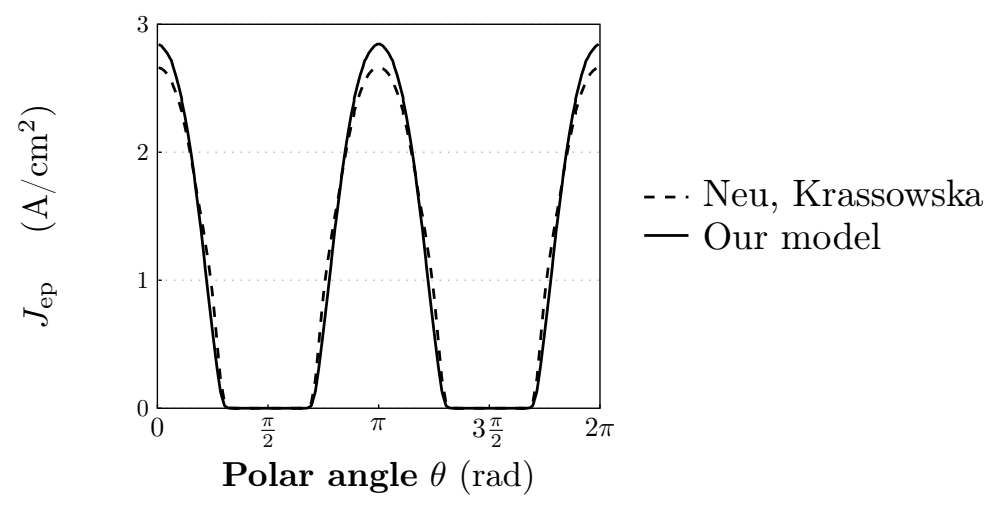

Figure 9: Current density through the membrane of the model of Neu, Krassowska, et al., that is $J_{\mathrm{ep}}=N_{\mathrm{ep}} i_{\mathrm{ep}}$ (in dashed line), compared with the membrane current density of our model (in solid line), $J_{\mathrm{ep}}=\widetilde{S}_{\mathrm{m}}[U]$, along the the cell membrane at $100 \mu \mathrm{s}$.

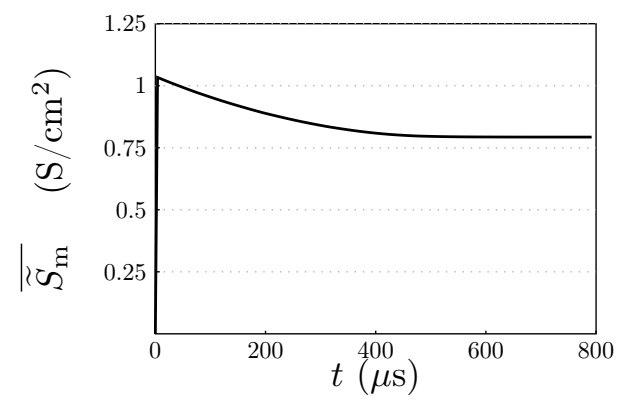

Figure 10: Averaged membrane conductivity $\widetilde{S}_{\mathrm{m}}$ during long-time simulations. Steady state is reached after several hundreds of microseconds, which is larger than usual pulse duration. 
uniqueness results. We then derive a new dynamic model of cell electropermeabilization, which takes the permeabilizing time into account. We studied mathematical properties of this new model. We then provided an accurate finite-difference method on cartesian grid to compute these models, and we eventually presented numerical simulations for both static and dynamic models, that corroborate the results of the most achieved model of Neu, Krassowska, et al.

The main feature of our models lies in the fact that without loss of accuracy it is composed by a small number of parameters (mainly 4 parameters: $S_{\mathrm{ir}}, V_{\mathrm{rev}}$, and $\tau_{\mathrm{ep}}$, and $\tau_{\text {res }}$ for the dynamical system) compared with the sophisticated models with tens of hardly measurable parameters of Neu, Krassowska, et al. Therefore, a forthcoming fitting of our models with the experimental data seems feasible, which is hardly the case for models with a large number of parameters.

From the biological point of view, we highlight the fact that the static model can be used for very long pulses (around $1 \mathrm{~ms}$ ) but, for short pulses around $10 \mu$ s and below, the dynamics of the phenomenon have to be considered.

\section{Acknowledgements}

The authors thank very warmly the biologists of the CNRS research team "UMR 8203 Vectorology and anti-cancerous therapeutics" at the Institut Gustave Roussy. Particulary, the discussions with Dr. A. Silve and Dr. L.M. Mir were very helpful in the understanding of the cell electropermeabilization. This research has been partly granted by the French national agency throughout the research projects INTCELL (2010-BLAN-916-04) and MEMOVE (2011-BS01-006-01).

\section{References}

[1] F.M. André and J. Gehl et al. Efficiency of high - and low - voltage pulse combinations for gene electrotransfert in mucle, liver tumor and skin. Human Gene Therapy, 19, 2008.

[2] M. Cisternino and L. Weynans. A parallel second order cartesian method for elliptic interface problems. Commun. Comput. Phys., 12, 2012.

[3] K. DeBruin and W. Krassowska. Modelling electroporation in a single cell. I. Effects of field strength and rest potential. Biophysical Journal, 77:1213-1224, Sept 1999.

[4] K. DeBruin and W. Krassowska. Modelling electroporation in a single cell. II. Effects of ionic concentrations. Biophysical Journal, 77:1225-1233, Sept 1999.

[5] D.E. Goldman. Potential, impedance and rectification in membranes. J. General Physiology, $27: 37-60,1943$.

[6] T. R. Gowrishankar, A. T. Esser, Z. Vasilkoski, K. C. Smith, and J. C. Weaver. Microdosimetry for conventional and supra-electroporation in cells with organelles. Biochem Biophys Res Commun, 341(4):1266-1276, Mar 2006.

[7] A.L Hodgkin and B. Katz. The effect of sodium ions on the electrical activity of the giant squid axon. J.Physiology, 108:37-77, 1949.

[8] L. Hodgkin and P. Horowicz. The influence of potassium and chloride ions on the membrane potential of single muscle fibres. J. Physiol., 148:127-160, 1959.

[9] L. Hodgkin and A. Huxley. A quantitative description of membrane current and its application to conduction and excitation in nerve. J. Physiol., 117:500-544, 1952. 
[10] A. Ivorra, J. Villemejane, and L.M. Mir. Electrical modeling of the influence of medium conductivity on electroporation. Physical Chemistry Chemical Physics, 12(34):10055-10064, 2010 .

[11] M. Marty, G. Sersa, and J.-R. Garbay et al. Electrochemotherapy - an easy, highly effective and safe treatment of cutaneous and subcutaneous metastases: Results of esope (european standard operating procedures of electrochemotherapy) study. E.J.C Supplements, 4:3-13, 2006 .

[12] L.M. Mir. Therapeutic perspectives of in vivo cell electropermeabilization. Bioelectrochemistry, 53:1-10, 2001.

[13] L.M. Mir. Electroporation of cells in tissues. Methods for detecting cell electropermeabilisation in vivo. In Electroporation based Technologies and Treatment: proceedings of the international scientific workshop and postgraduate course, pages 32-35, 14-20 November 2005. Ljubljana, SLOVENIA.

[14] J. Neu and W. Krassowska. Asymptotic model of electroporation. Physical Review E, 53(3):3471-3482, Mar 1999.

[15] J. Neu and W. Krassowska. Singular perturbation analysis of the pore creation transient. Physical Review E, 74(031917):1-9, Sep 2006.

[16] S. Osher and J. A. Sethian. Fronts propagating with curvature-dependent speed: Algorithms based on hamilton-jacobi formulations. J. Comput. Phys., 79(12), 1988.

[17] M. Pavlin, T. Kotnik, D. Miklavcic, P. Kramar, and A.M. Lebar. Electroporation of planar lipid bilayers and membranes. Advanced in Planar Lipid Bilayers and Liposomes, 6(7), 2008.

[18] R. Perrussel and C. Poignard. Asymptotic expansion of steady-state potential in a high contrast medium with a thin resistive layer. INRIA research report RR-7163. http://hal.inria.fr/inria-00442659/fr/, 2011.

[19] G. Pucihar, T. Kotnik, B. Valič, and D. Miklavčič. Numerical determination of transmembrane voltage induced on irregularly shaped cells. Ann Biomed Eng, 34(4):642-652, Apr 2006.

[20] G. Serša. Application of electroporation in electrochemotherapy of tumors. In Electroporation based Technologies and Treatment: proceedings of the international scientific workshop and postgraduate course, pages 42-45, 14-20 November 2005. Ljubljana, SLOVENIA.

[21] J. Smith, K. Neu and W. Krassowska. Model of creation and evolution of stable electropores for DNA delivery. Biophys. J., 86:2813-2826, May 2004.

[22] J. Teissié. In Vitro cell electropermeabilization. In Electroporation based Technologies and Treatment: proceedings of the international scientific workshop and postgraduate course, pages 29-31, 14-20 November 2005. Ljubljana, SLOVENIA.

[23] J. Teissié, M. Golzio, and M.P. Rols. Mechanisms of cell membrane electropermeabilization: A minireview of our present (lack of?) knowledge. Biochimica et Biophysica Acta, 1724:270280,2005 . 


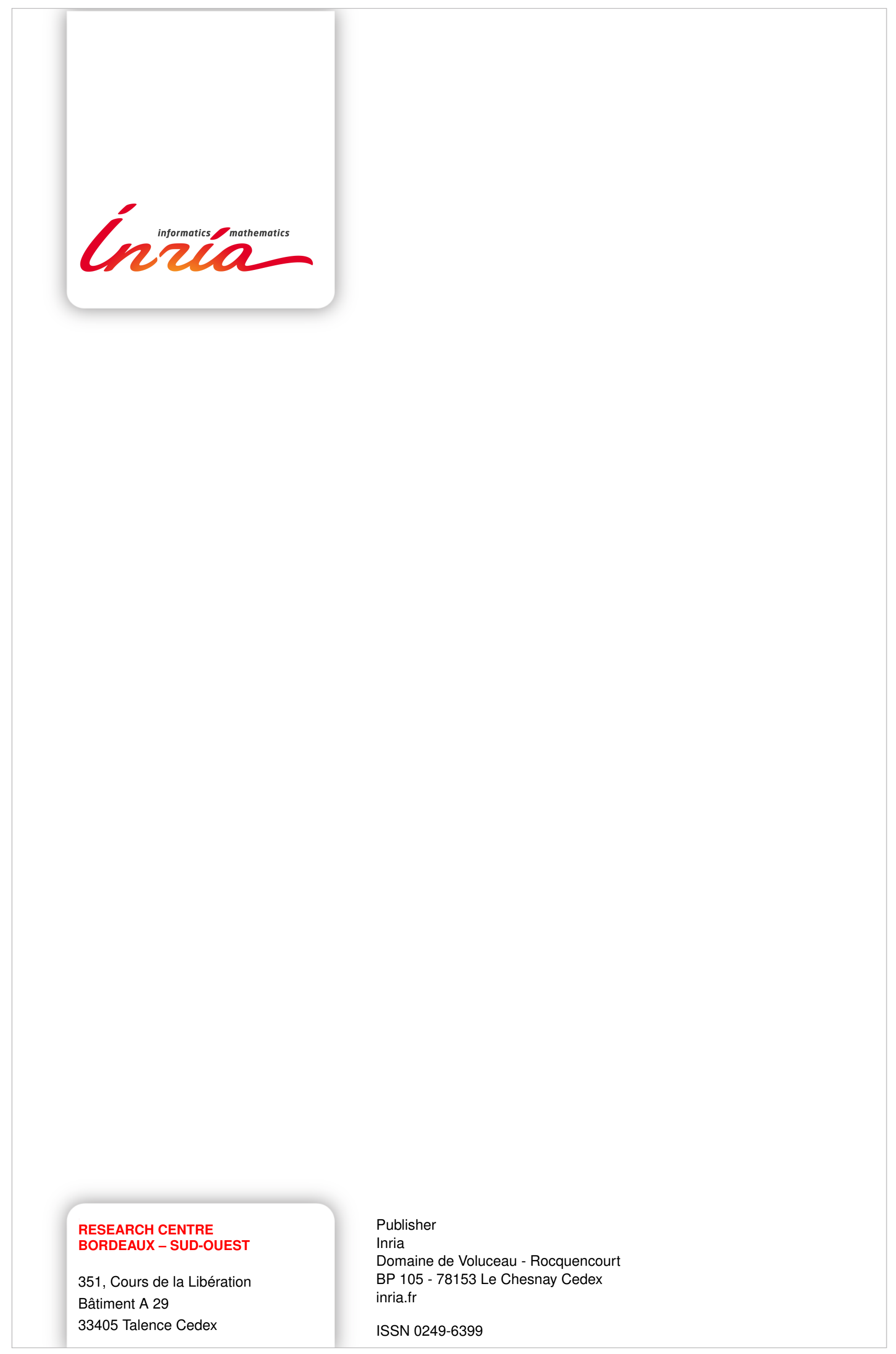

